\title{
Recent results from the hermes experiment
}

Charlotte Van Hulse, on behalf of the HERMES collaboration University of the Basque Country - UPV/EHU

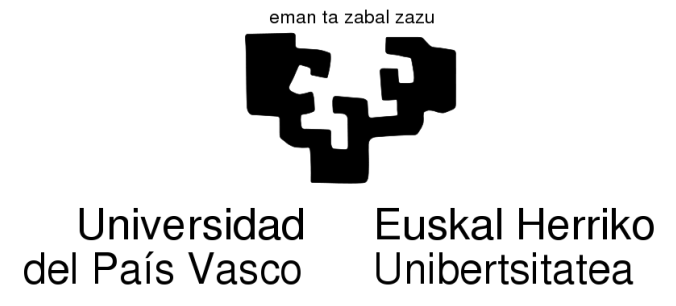

\section{ікеrbasque}

Basque Foundation for Science

PacSpin 2013, Ji'nan 


\section{The nucleon in multiple dimensions}

Wigner distributions $W\left(x, \vec{k}_{T}, \overrightarrow{b_{\perp}}\right)$

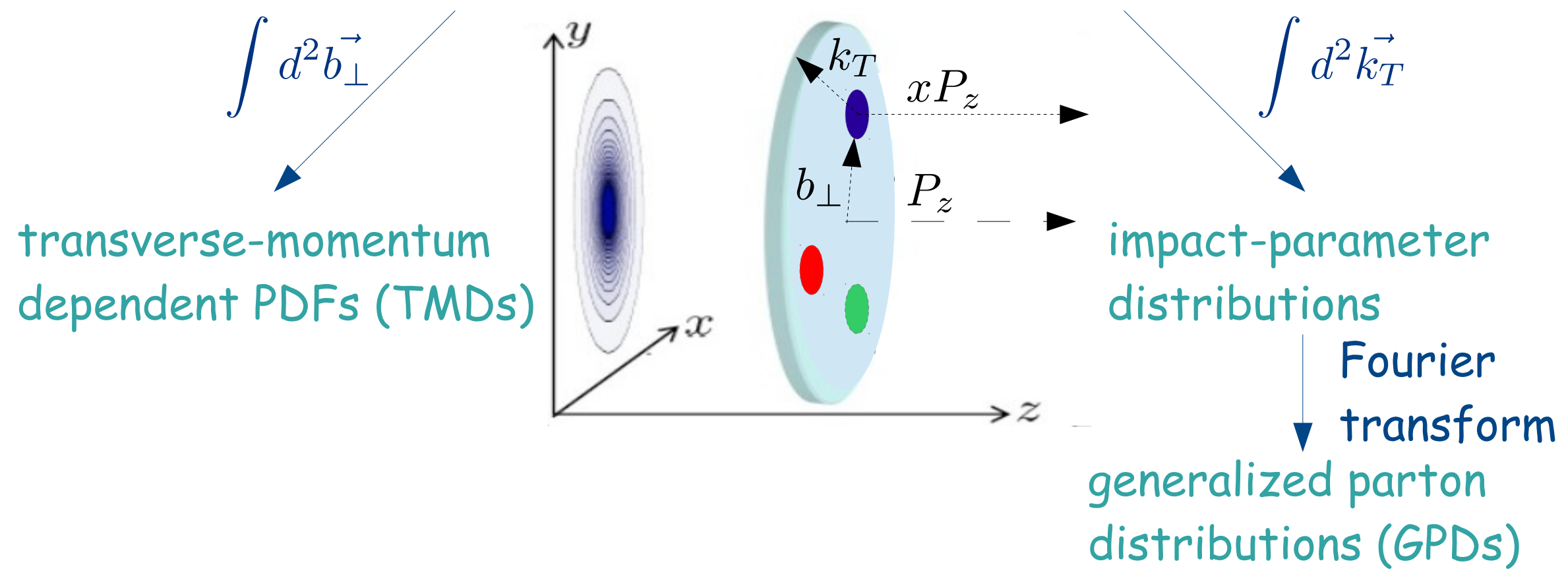




\section{The nucleon in multiple dimensions}

Wigner distributions $W\left(x, \vec{k}_{T}, \overrightarrow{b_{\perp}}\right)$

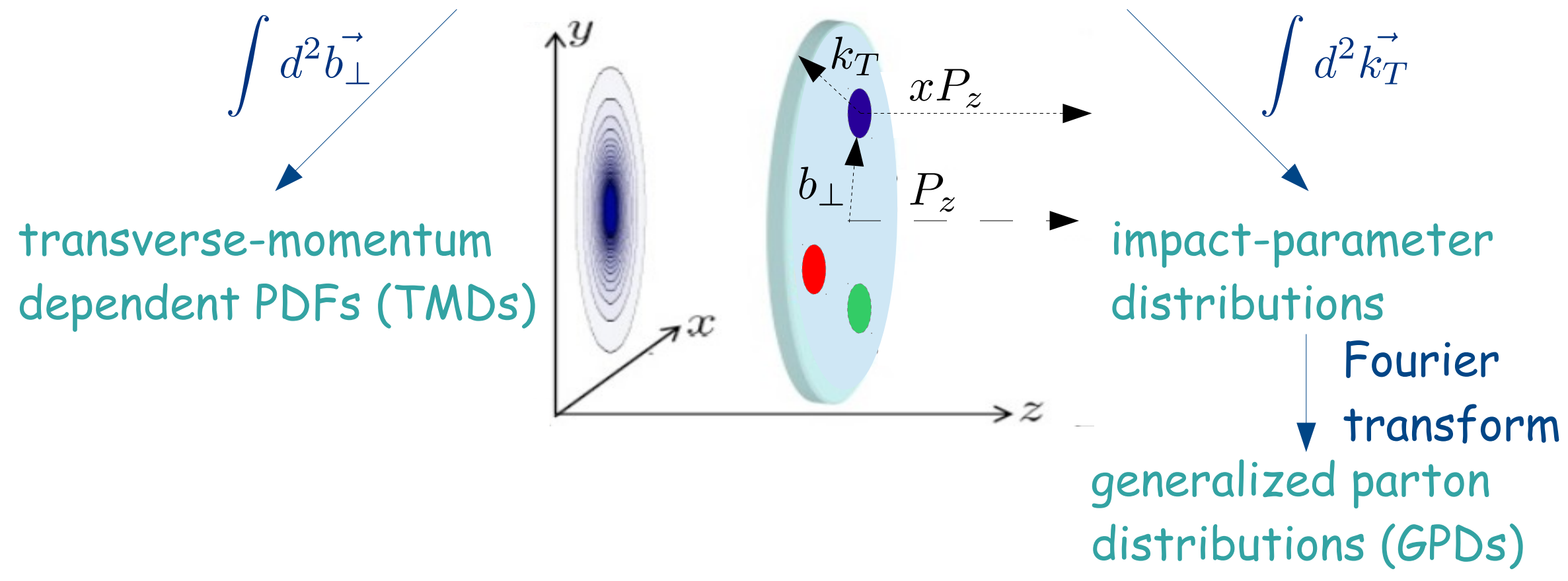

semi-inclusive deep-inelastic scattering (DIS) hard exclusive reactions
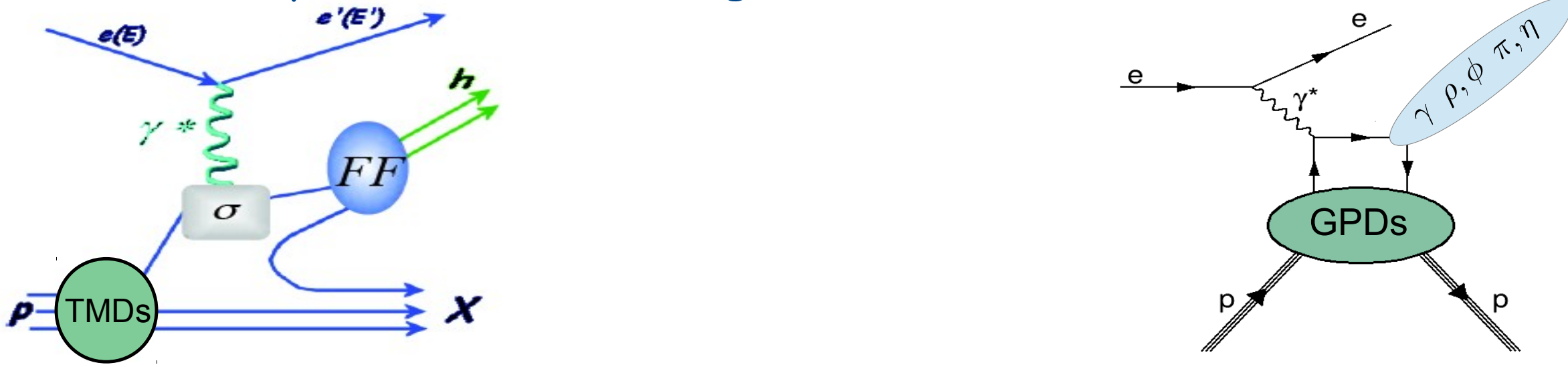
Semi-inclusive DIS 


\section{Semi-inclusive DIS cross section}

$\frac{d \sigma}{d x d y d z d \phi_{h} d P_{h \perp}^{2} d \phi_{S}}=\frac{\alpha^{2}}{x y Q^{2}} \frac{y^{2}}{2(1-\epsilon)}\left(1+\frac{\gamma^{2}}{2 x}\right)$

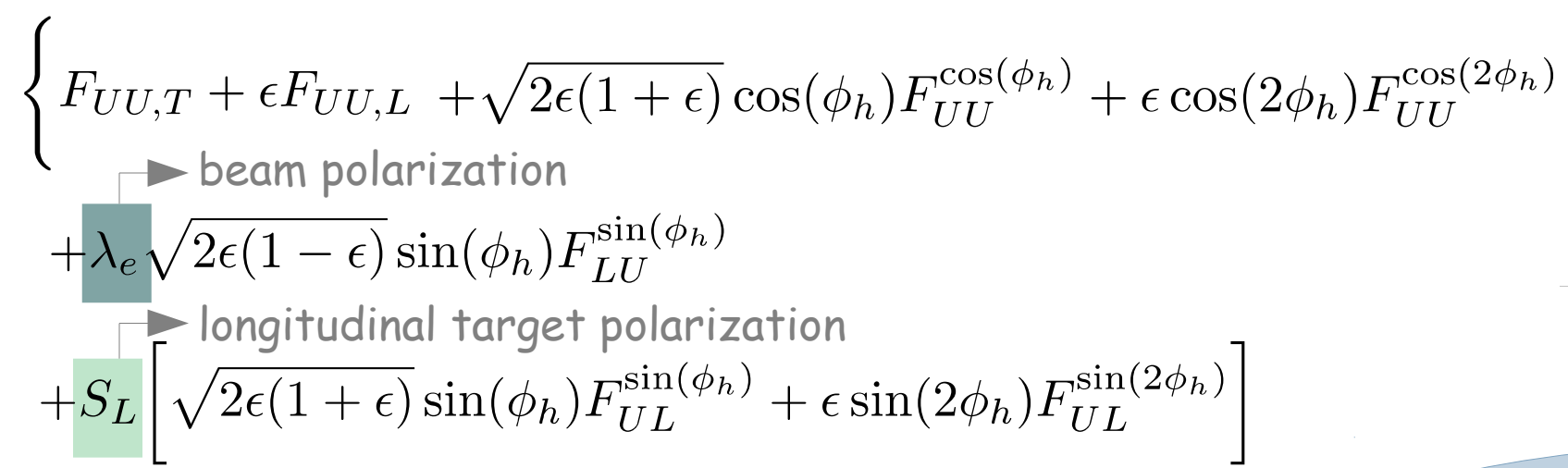

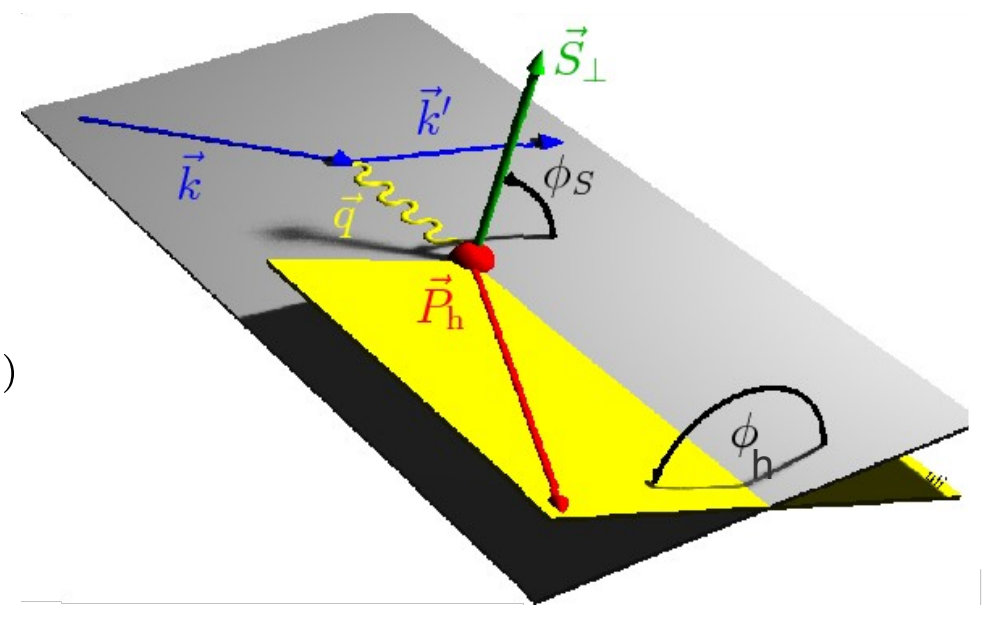

$+S_{L} \lambda_{e}\left[\sqrt{1-\epsilon^{2}} F_{L L}+\sqrt{2 \epsilon(1-\epsilon)} \cos \left(\phi_{h}\right) F_{L L}^{\cos \left(\phi_{h}\right)}\right]$

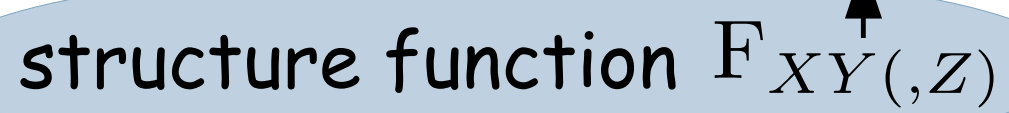

transverse target polarization

$+S_{T}\left[\sin \left(\phi_{h}-\phi_{S}\right)\left(F_{U T, T}^{\sin \left(\phi_{h}-\phi_{S}\right)}+\epsilon F_{U T, L}^{\sin \left(\phi_{h}-\phi_{S}\right)}\right)\right.$

target

polarization

$+\epsilon \sin \left(\phi_{h}+\phi_{S}\right) F_{U T}^{\sin \left(\phi_{h}+\phi_{S}\right)}+\epsilon \sin \left(3 \phi_{h}-\phi_{S}\right) F_{U T}^{\sin \left(3 \phi_{h}-\phi_{S}\right)}$

$\left.+\sqrt{2 \epsilon(1+\epsilon)} \sin \left(\phi_{S}\right) F_{U T}^{\sin \left(\phi_{S}\right)}+\sqrt{2 \epsilon(1+\epsilon)} \sin \left(2 \phi_{h}-\phi_{S}\right) F_{U T}^{\sin \left(2 \phi_{h}-\phi_{S}\right)}\right]$

$+S_{T} \lambda_{e}\left[\sqrt{1-\epsilon^{2}} \cos \left(\phi_{h}-\phi_{S}\right) F_{L T}^{\cos \left(\phi_{h}-\phi_{S}\right)}+\sqrt{2 \epsilon(1-\epsilon)} \cos \left(\phi_{S}\right) F_{L T}^{\cos \left(\phi_{S}\right)}\right.$

$\left.\left.+\quad \sqrt{2 \epsilon(1-\epsilon)} \cos \left(2 \phi_{h}-\phi_{S}\right) F_{L T}^{\cos \left(2 \phi_{h}-\phi_{S}\right)}\right]\right\}$ 


\section{Semi-inclusive DIS cross section}

$\frac{d \sigma}{d x d y d z d \phi_{h} d P_{h \perp}^{2} d \phi_{S}}=\frac{\alpha^{2}}{x y Q^{2}} \frac{y^{2}}{2(1-\epsilon)}\left(1+\frac{\gamma^{2}}{2 x}\right)$

$\left\{F_{U U, T}+\epsilon F_{U U, L}+\sqrt{2 \epsilon(1+\epsilon)} \cos \left(\phi_{h}\right) F_{U U}^{\cos \left(\phi_{h}\right)}+\epsilon \cos \left(2 \phi_{h} F_{U U}^{\cos \left(2 \phi_{h}\right)}\right.\right.$ - beam polarization

$+\lambda_{e} \sqrt{2 \epsilon(1-\epsilon)} \sin \left(\phi_{h}\right) F_{L U}^{\sin \left(\phi_{h}\right)}$

longitudinal target polarization

$+S_{L}\left[\sqrt{2 \epsilon(1+\epsilon)} \sin \left(\phi_{h}\right) F_{U L}^{\sin \left(\phi_{h}\right)}+\epsilon \sin \left(2 \phi_{h} F_{U L}^{\sin \left(2 \phi_{h}\right)}\right.\right.$

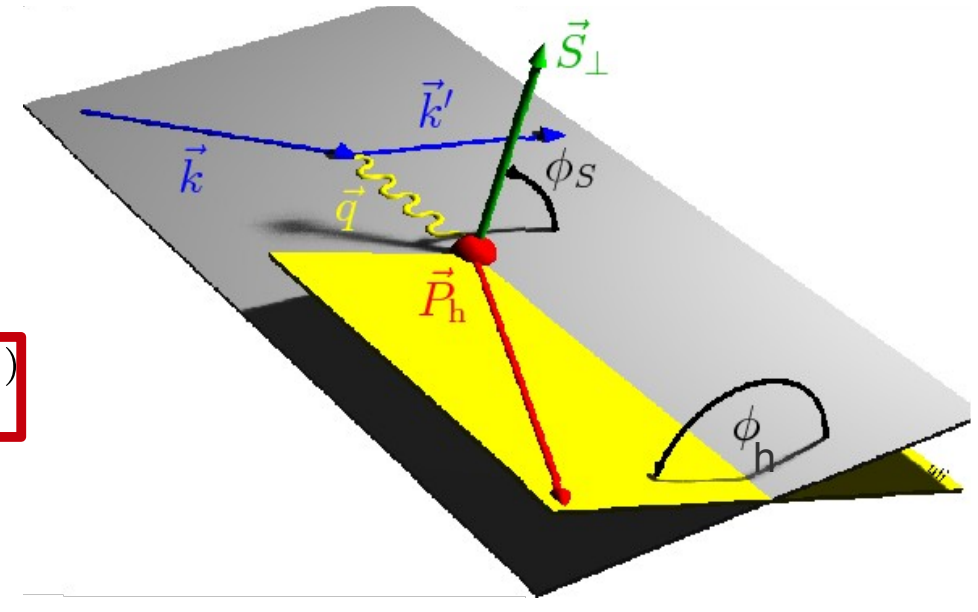

$+S_{L} \lambda_{e}\left[\sqrt{1-\epsilon^{2} F_{L L}}+\sqrt{2 \epsilon(1-\epsilon)} \cos \left(\phi_{h}\right) F_{L L}^{\cos \left(\phi_{h}\right)}\right]$

$\rightarrow$ transverse target polarization

$+S_{T}\left[\sin \left(\phi_{h}-\phi_{S}\right) F_{U T, T}^{\sin \left(\phi_{h}-\phi_{S}\right)}+\epsilon F_{U T, L}^{\sin \left(\phi_{h}-\phi_{S}\right)}\right.$

$+\epsilon \sin \left(\phi_{h}+\phi_{S} F_{U T}^{\sin \left(\phi_{h}+\phi_{S}\right)}+\epsilon \sin \left(3 \phi_{h}-\phi_{S} F_{U T}^{\sin \left(3 \phi_{h}-\phi_{S}\right)}\right.\right.$

$\left.+\sqrt{2 \epsilon(1+\epsilon)} \sin \left(\phi_{S}\right) F_{U T}^{\sin \left(\phi_{S}\right)}+\sqrt{2 \epsilon(1+\epsilon)} \sin \left(2 \phi_{h}-\phi_{S}\right) F_{U T}^{\sin \left(2 \phi_{h}-\phi_{S}\right)}\right]$

$+S_{T} \lambda_{e}\left[\sqrt{1-\epsilon^{2}} \cos \left(\phi_{h}-\phi_{S}{ }_{F_{L T}^{\cos \left(\phi_{h}-\phi_{S}\right)}}+\sqrt{2 \epsilon(1-\epsilon)} \cos \left(\phi_{S}\right) F_{L T}^{\cos \left(\phi_{S}\right)}\right.\right.$

$\left.\left.+\quad \sqrt{2 \epsilon(1-\epsilon)} \cos \left(2 \phi_{h}-\phi_{S}\right) F_{L T}^{\cos \left(2 \phi_{h}-\phi_{S}\right)}\right]\right\}$

target

polarization

structure function $\mathrm{F}_{X Y(, Z)}$

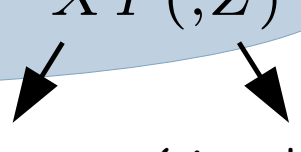

beam (virtual photon polarization polarization)

leading twist 


\section{Semi-inclusive DIS cross section}

structure function $F_{X Y} \propto T M D \otimes F F$

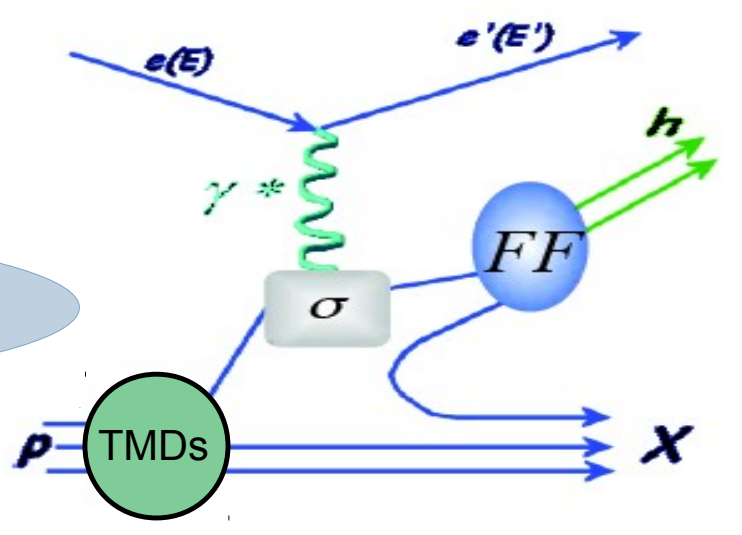

transverse momentum distributions (TMDs)

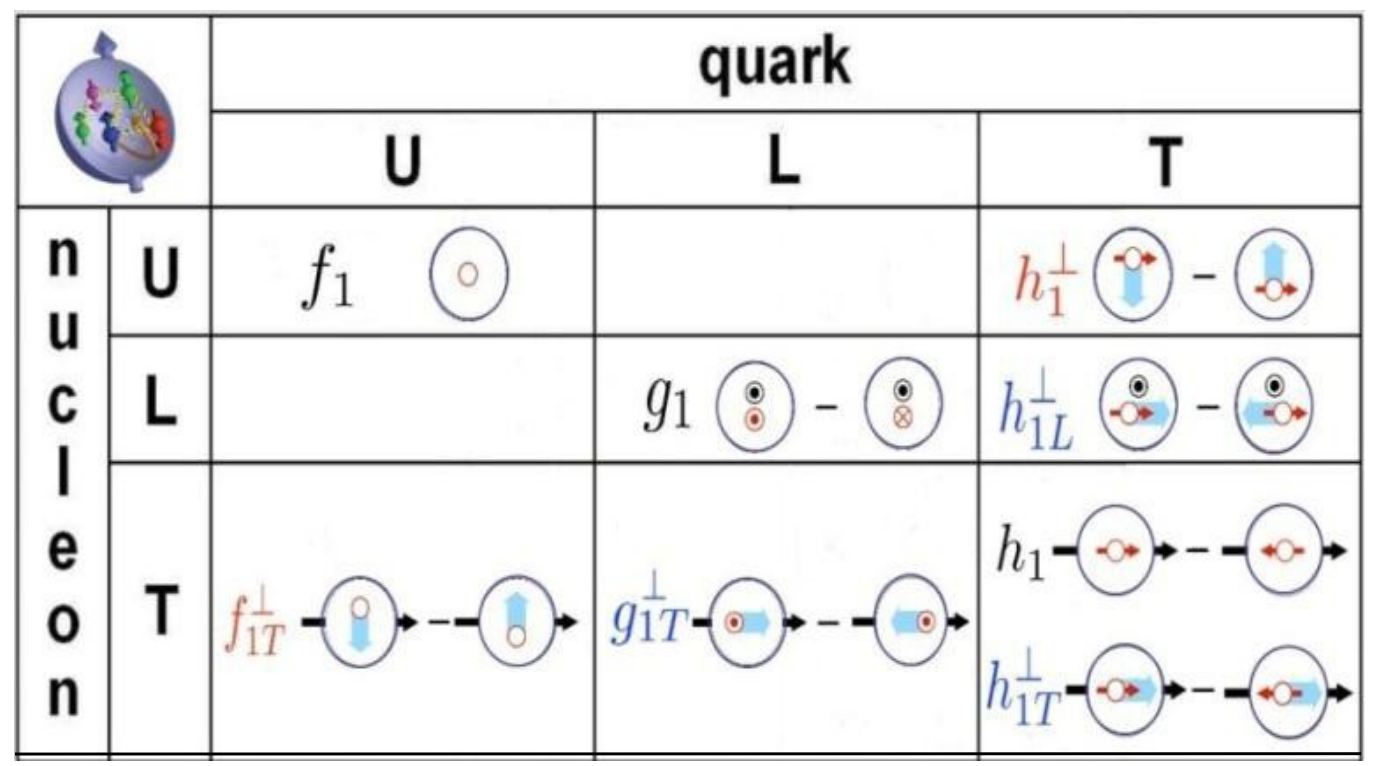

$\rightarrow$ (0) nucleon with transverse/longitudinal spin

$\rightarrow \quad$ quark with transverse/longitudinal spin fragmentation functions (FFs)

\begin{tabular}{|c|c|c|c|}
\hline & \multicolumn{3}{|c|}{ quark } \\
\hline & U & $L$ & $T$ \\
\hline $\mathrm{h}$ & $D_{1} \odot$ & & $H_{1}^{\perp} \infty-\infty$ \\
\hline
\end{tabular}




\section{Semi-inclusive DIS cross section}

structure function $F_{X Y} \propto T M D \otimes F F$

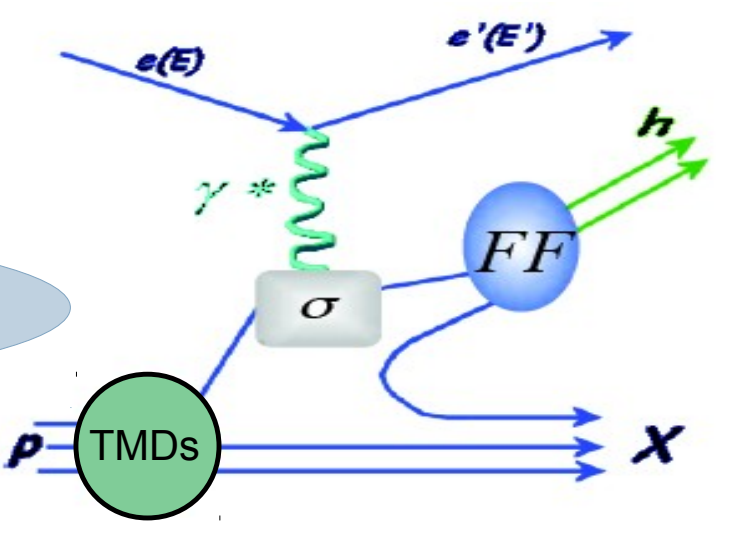

transverse momentum distributions (TMDs)

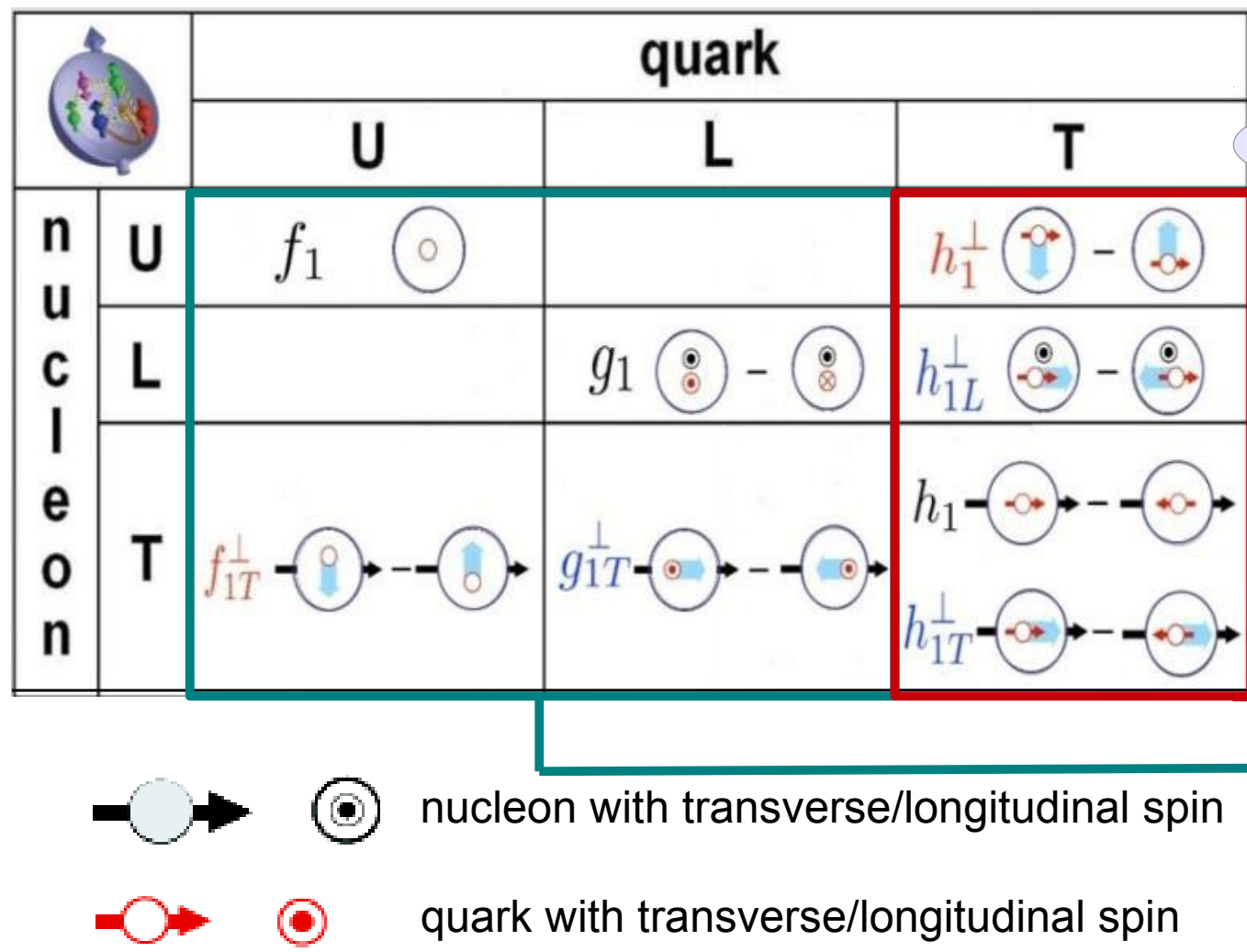

fragmentation functions (FFs)

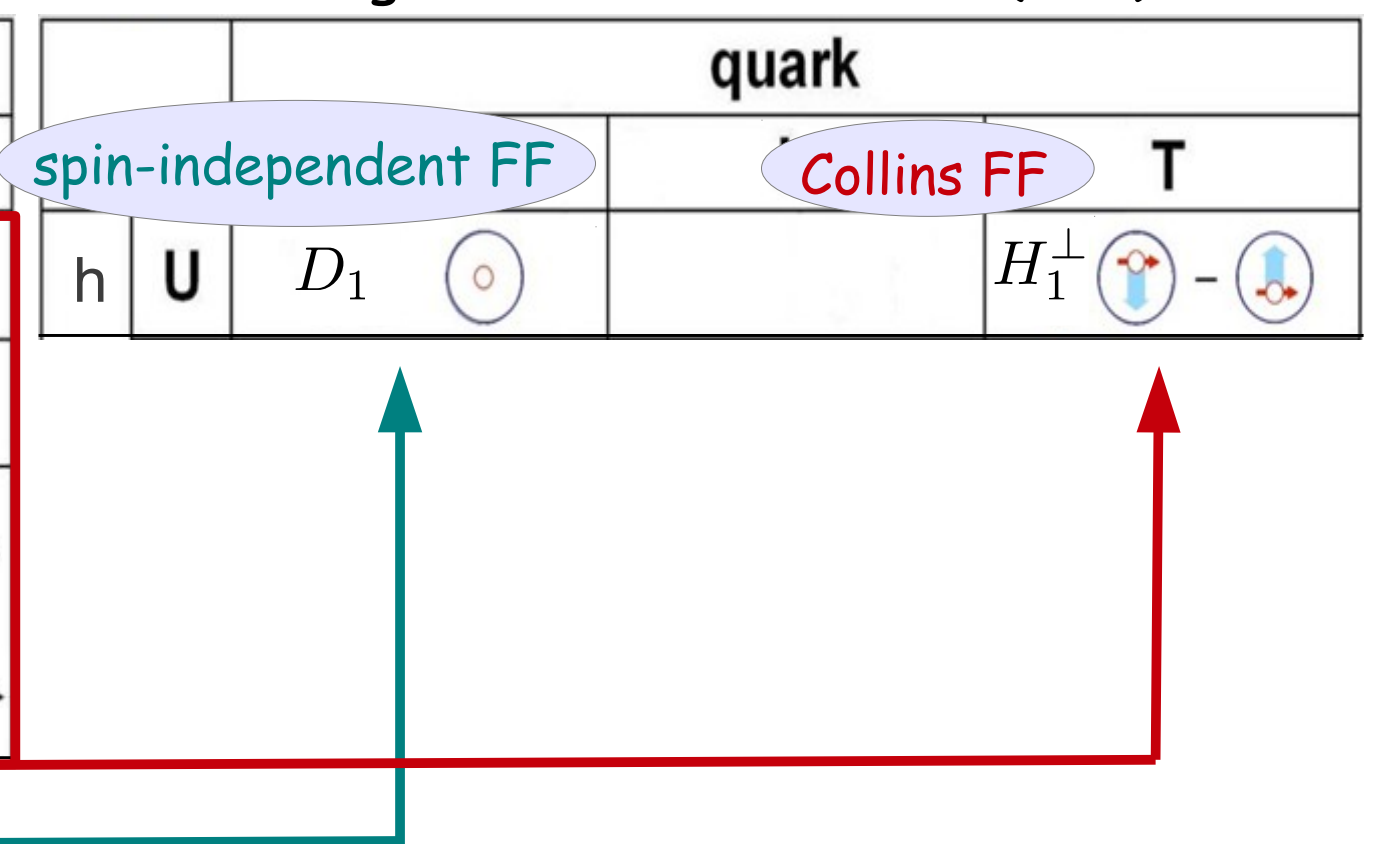




\section{Semi-inclusive DIS cross section}

structure function $F_{X Y} \propto T M D \otimes F F$

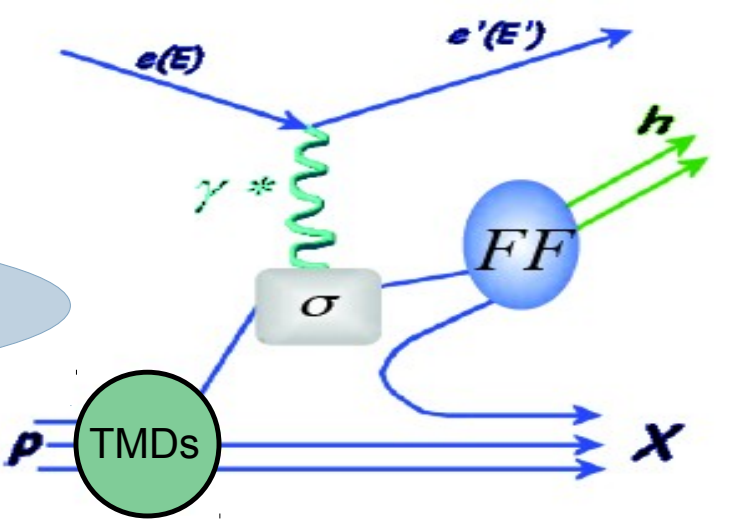

transverse momentum distributions (TMDs)

\begin{tabular}{|c|c|c|}
\hline & \multicolumn{2}{|c|}{ quark } \\
\hline spin-independent DF & Boer-Mulders DF T \\
\hline
\end{tabular}

.
n

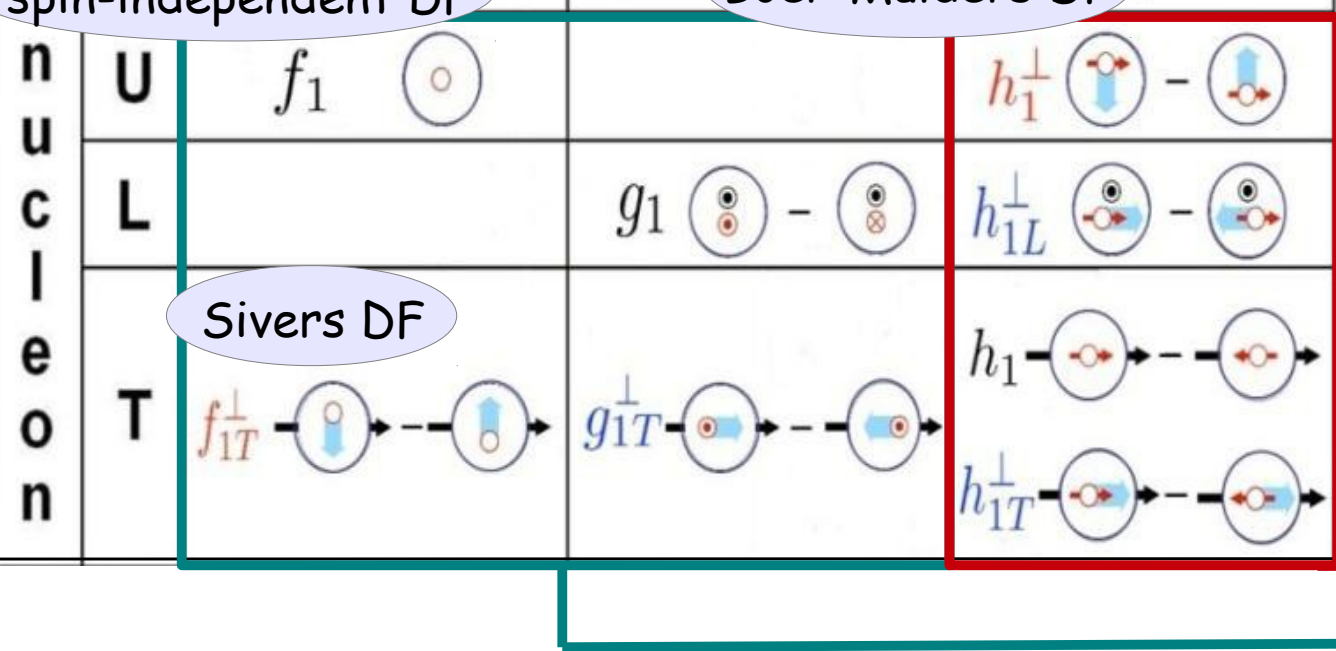

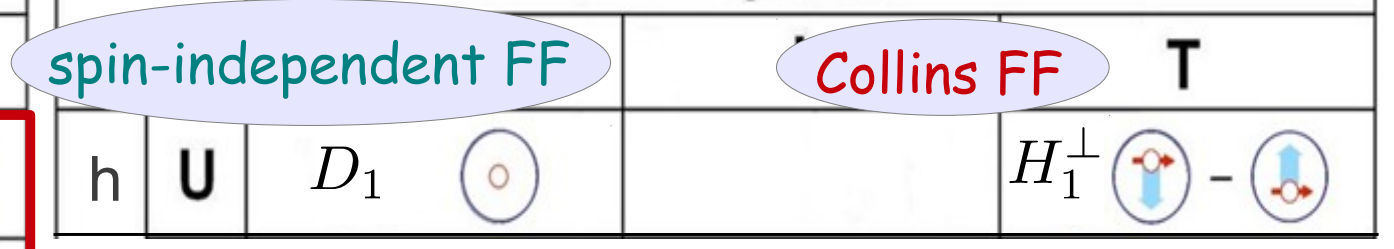

$\rightarrow$ (0) nucleon with transverse/longitudinal spin

$\rightarrow \quad$ quark with transverse/longitudinal spin 
Hadron multiplicities 


\section{Hadron multiplicities}

$$
\begin{aligned}
\frac{d \sigma}{d x d y d z d \phi_{h} d P_{h \perp}^{2}} & \int d d \phi_{h} \\
\mathrm{M}^{h}\left(x_{B}, Q^{2}, z, P_{h \perp}\right)= & \frac{1}{d^{2} N^{D I S}\left(x_{B}, Q^{2}\right)} \frac{d^{4} N^{h}\left(x_{B}, Q^{2}, z, P_{h \perp}\right)}{d z d P_{h \perp}} \\
& \propto \frac{F_{U U, T}+\epsilon F_{U U, L}}{F_{T}+\epsilon F_{L}} \\
& \propto \frac{\sum_{q} e_{q}^{2} f_{1}^{q}\left(x_{B}, k_{T}^{2}, Q^{2}\right) \otimes D_{1}^{q}\left(z, p_{T}^{2}, Q^{2}\right)}{\sum_{q} e_{q}^{2} f_{1}^{q}\left(x_{B}, Q^{2}\right)}
\end{aligned}
$$

$k_{T}$ : transverse momentum of struck quark $p_{T}$ : transverse momentum of fragmenting quark 


\section{Results projected in $z$}

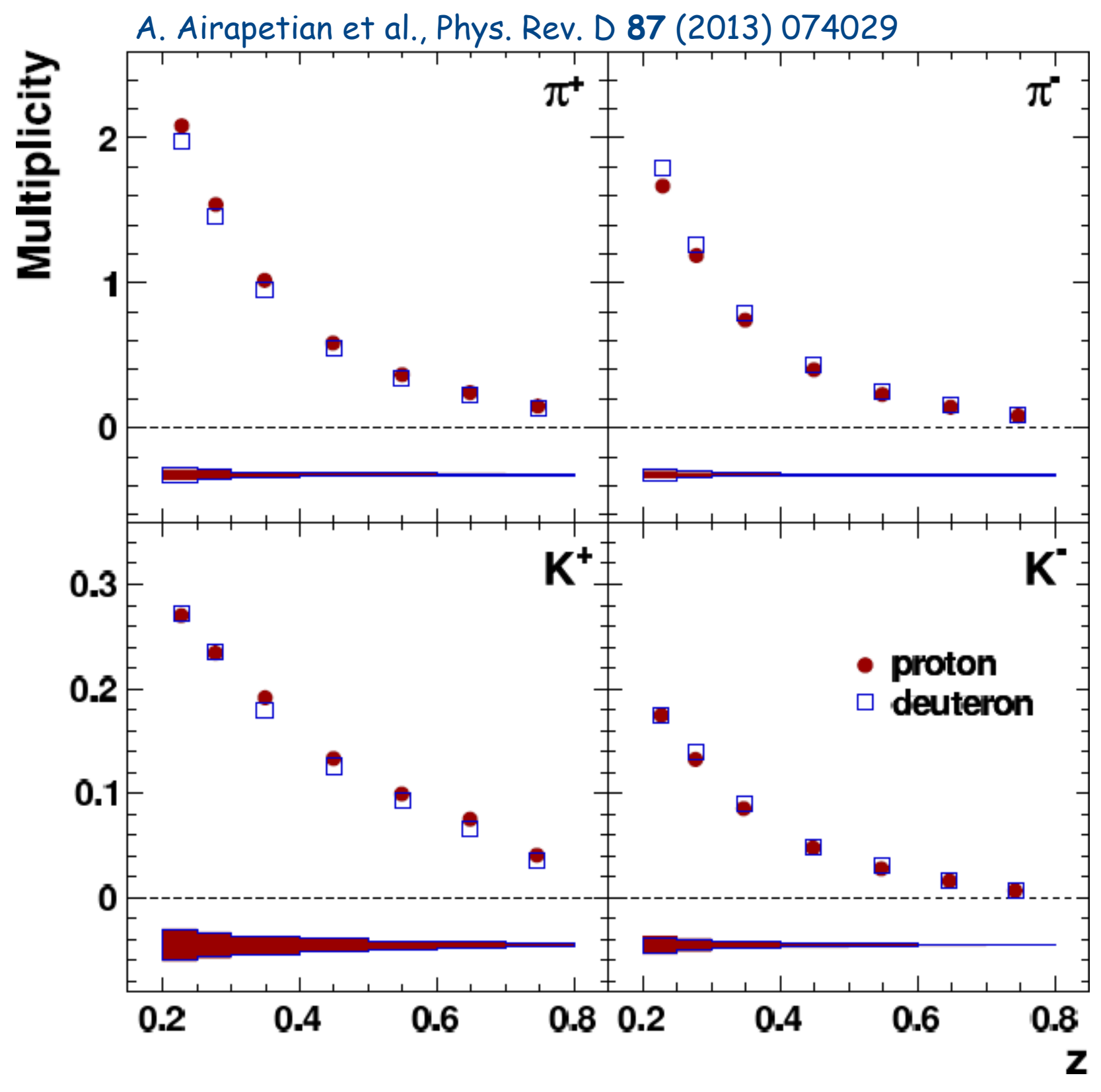

multiplicities reflect

- nucleon valence-quark content (u-dominance)

- favored $\leftrightarrow$ unfavored fragmentation 


\section{Comparison to models}

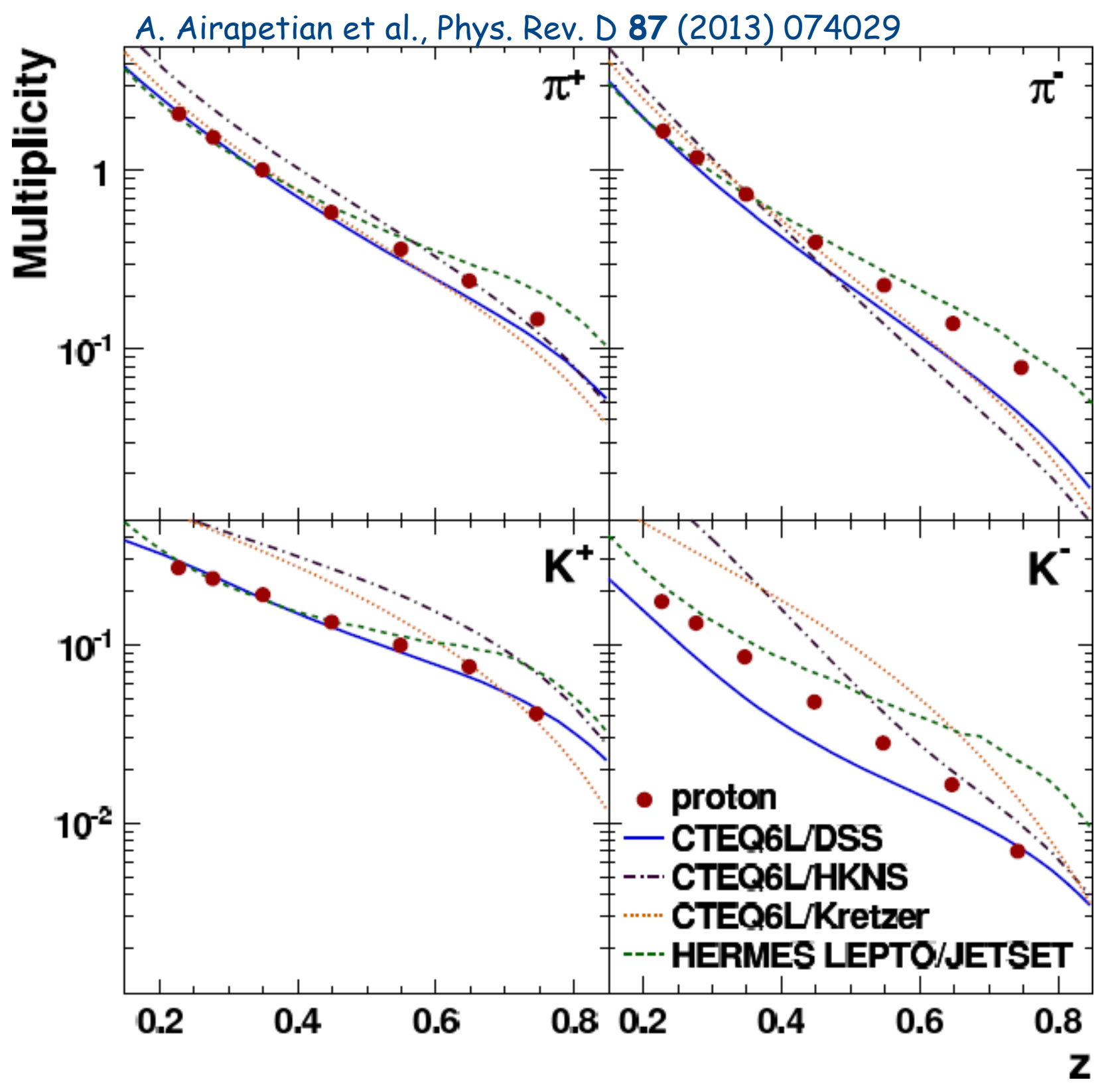

- LO in $\alpha_{S}$

- CTEQ6L PDFS JHEP 0207 (2002) 012

- DSS FFS Phys. Rev. D 75 (2007) 114010

- Kretzer FFs Phys. Rev. D 62 (2000) 054001

- reasonable agreement between DSS and positive mesons

- poor agreement for negative mesons 


\section{Results projected in $\mathrm{z}$ and $\mathrm{P}_{\mathrm{h} \perp}$}
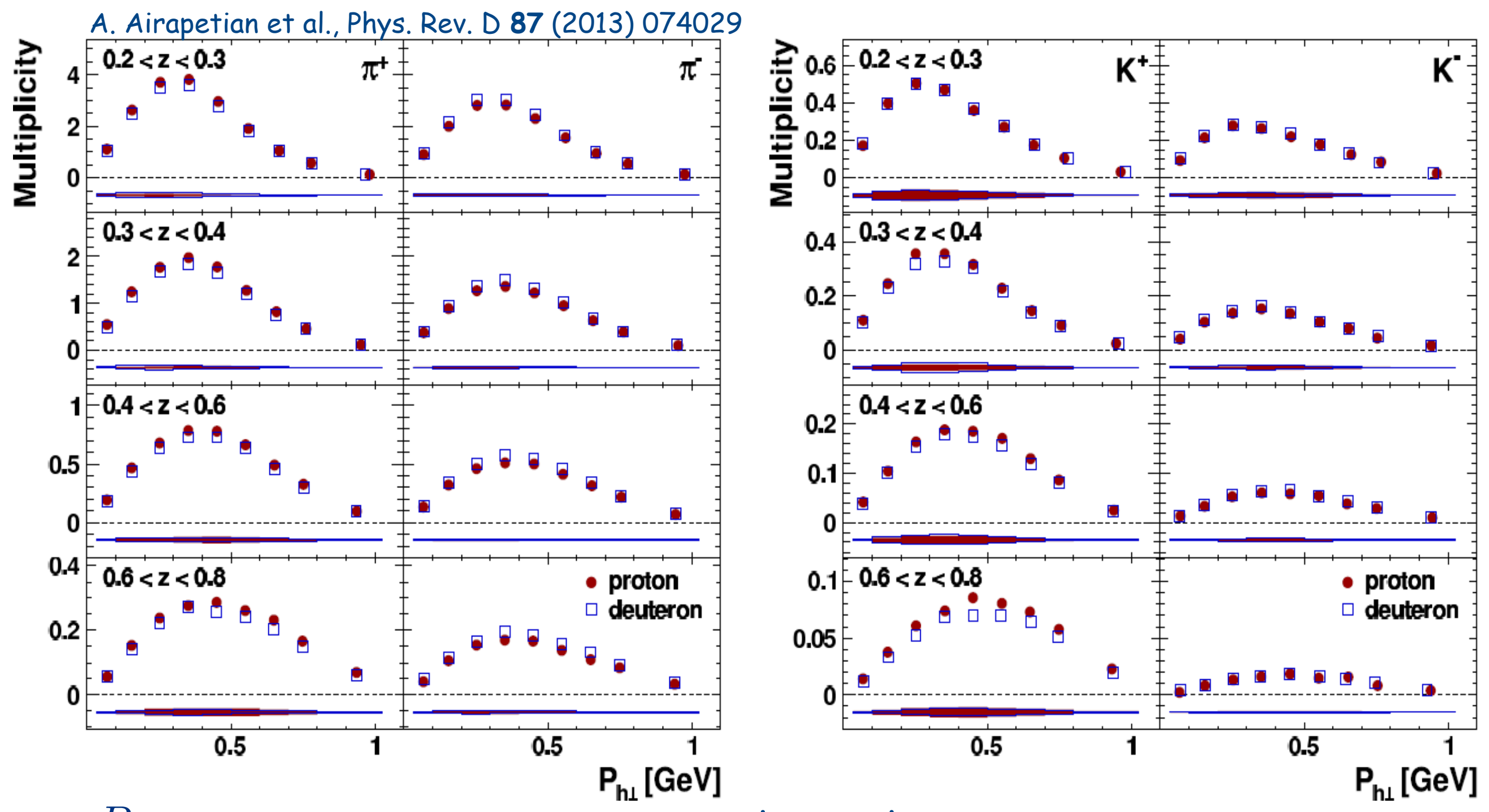

- $P_{h \perp}$ : - transverse intrinsic struck-quark momentum

- transverse momentum from fragmentation process

- $K^{-}:$broader distribution

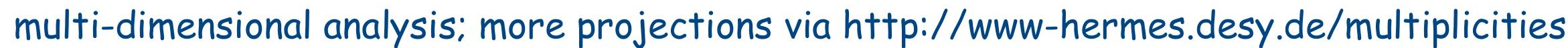




\section{Re-evaluation of the strange quark} distribution

$\mathrm{K}^{ \pm}$multiplicities from unpolarized deuterium

$$
\begin{array}{ll}
\frac{d^{2} N^{K}(x)}{d^{2} N^{D I S}(x)}=\frac{Q(x) \int_{0.2}^{0.8} \mathcal{D}_{Q}^{K}(z) d z+S(x) \int_{0.2}^{0.8} \mathcal{D}_{S}^{K}(z) d z}{5 Q(x)+2 S(x)} \\
Q(x) \equiv u(x)+\bar{u}(x)+d(x)+\bar{d}(x) & \mathcal{D}_{Q}^{K}(z) \equiv 4 D_{u}^{K}(z)+D_{d}^{K}(z) \\
S(x) \equiv s(x)+\bar{s}(x) & \mathcal{D}_{S}^{K}(z) \equiv 2 D_{s}^{K}(z)
\end{array}
$$




\section{Re-evaluation of the strange quark}

\section{distribution}

$\mathrm{K}^{ \pm}$multiplicities from unpolarized deuterium

$$
\begin{array}{ll}
\frac{d^{2} N^{K}(x)}{d^{2} N^{D I S}(x)}=\frac{Q(x) \int_{0.2}^{0.8} \mathcal{D}_{Q}^{K}(z) d z+S(x) \int_{0.2}^{0.8} \mathcal{D}_{S}^{K}(z) d z}{5 Q(x)+2 S(x)} \\
Q(x) \equiv u(x)+\bar{u}(x)+d(x)+\bar{d}(x) & \mathcal{D}_{Q}^{K}(z) \equiv 4 D_{u}^{K}(z)+D_{d}^{K}(z) \\
S(x) \equiv s(x)+\bar{s}(x) & \mathcal{D}_{S}^{K}(z) \equiv 2 D_{s}^{K}(z)
\end{array}
$$
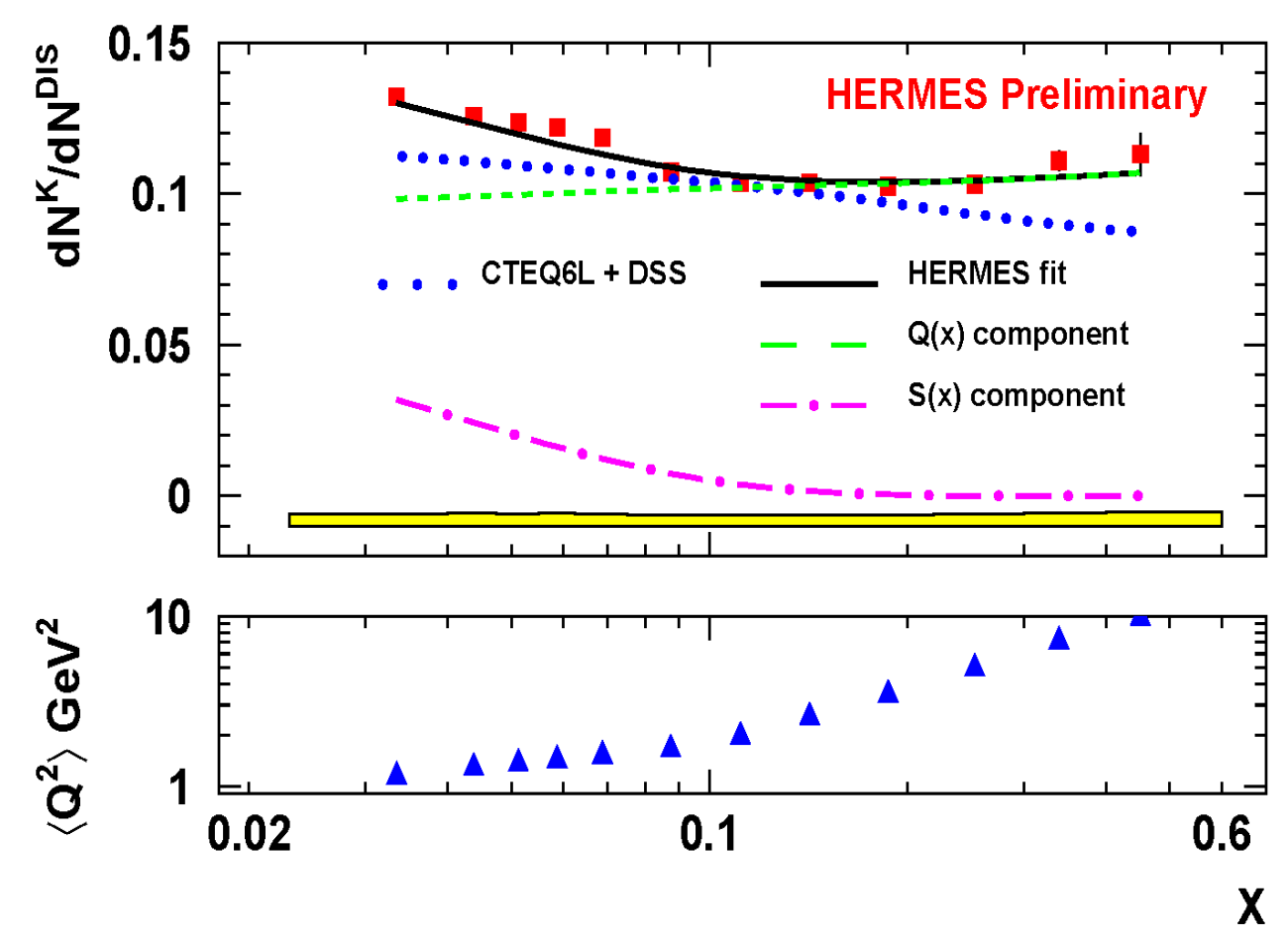

$S(x) \approx 0$ for $x>0.1$

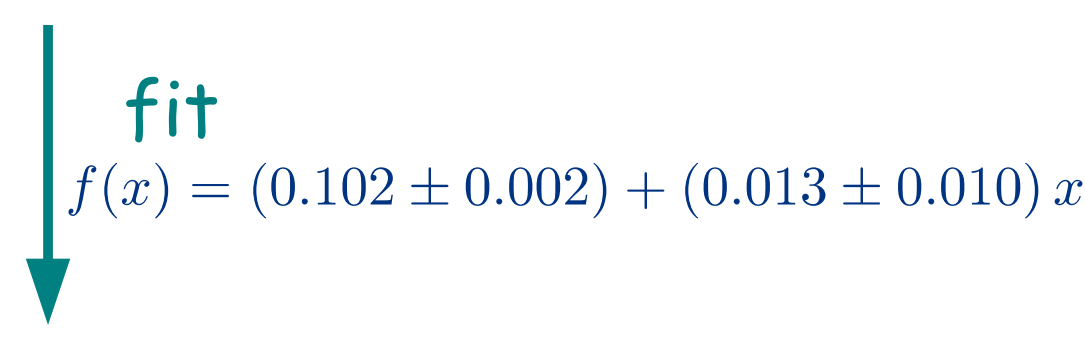

$$
\int_{0.2}^{0.8} \mathcal{D}_{Q}^{K}(z) d z=0.398 \pm 0.010
$$


Re-evaluation of the strange quark distribution

$Q(x)$ from CTEQ6L
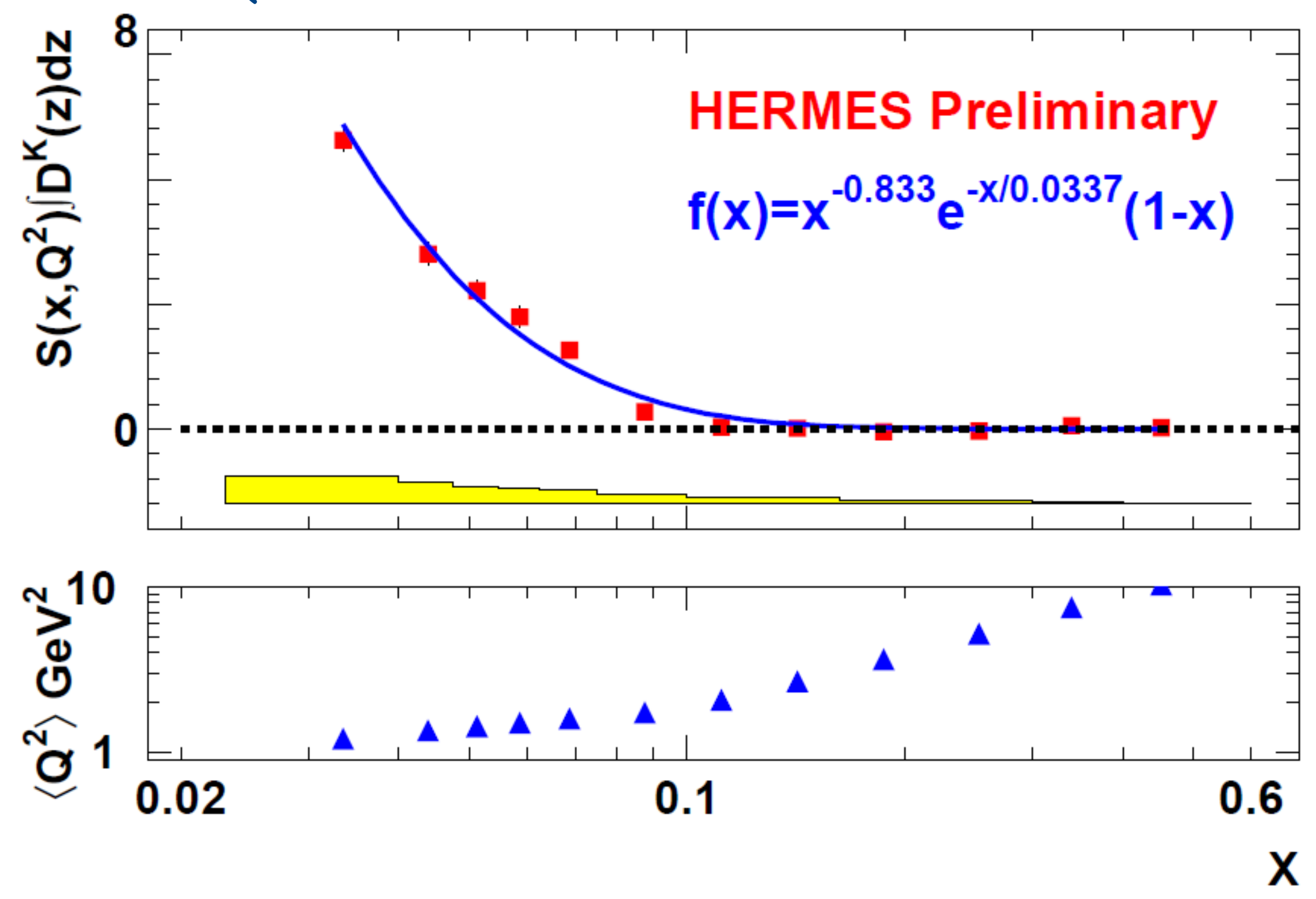


\section{Re-evaluation of the strange quark}

\section{distribution}

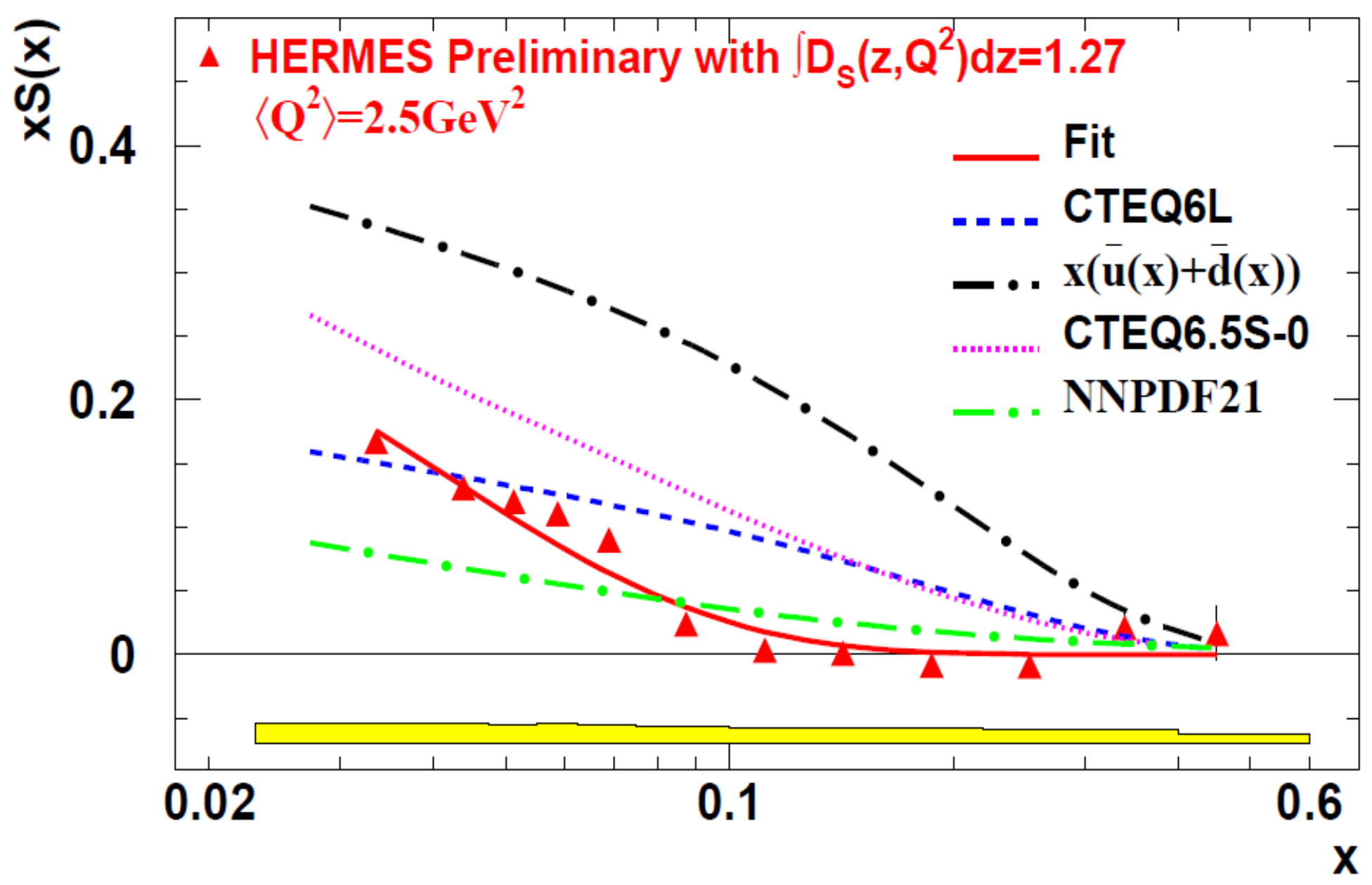

- $x S(x)$ for certain value of $\int \mathcal{D}_{S}^{K}(z) d z$

- independent of value, shape of $x S(x)$ incompatible with predictions 


\section{non-collinear semi-inclusive DIS}

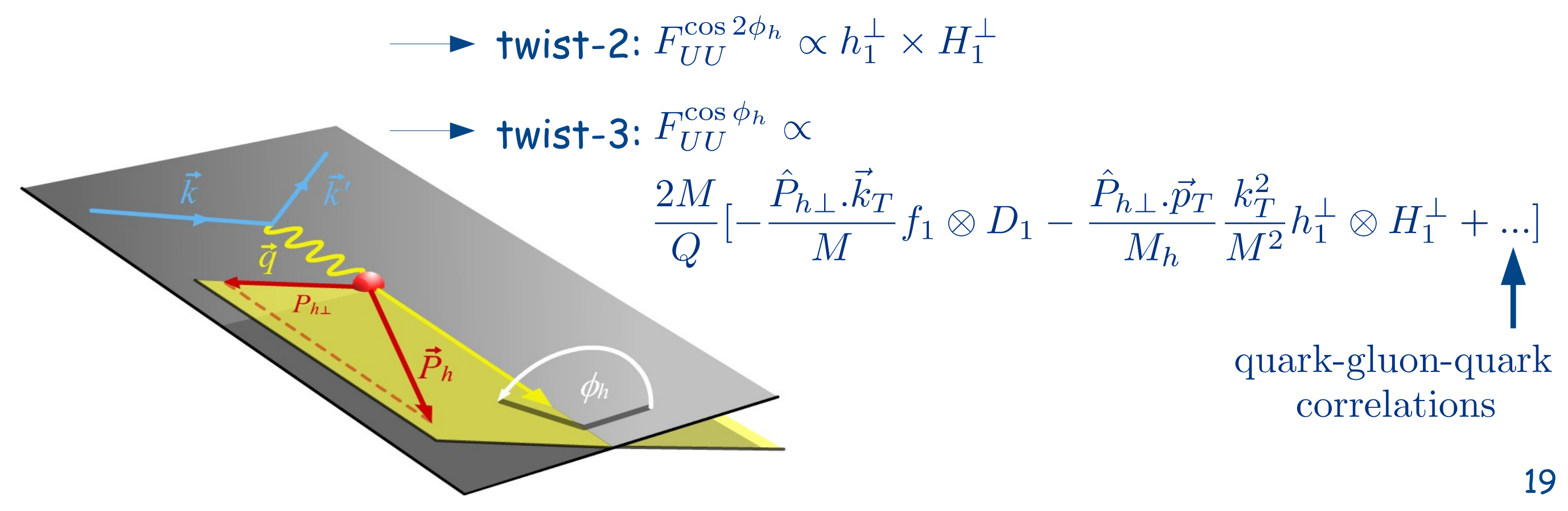




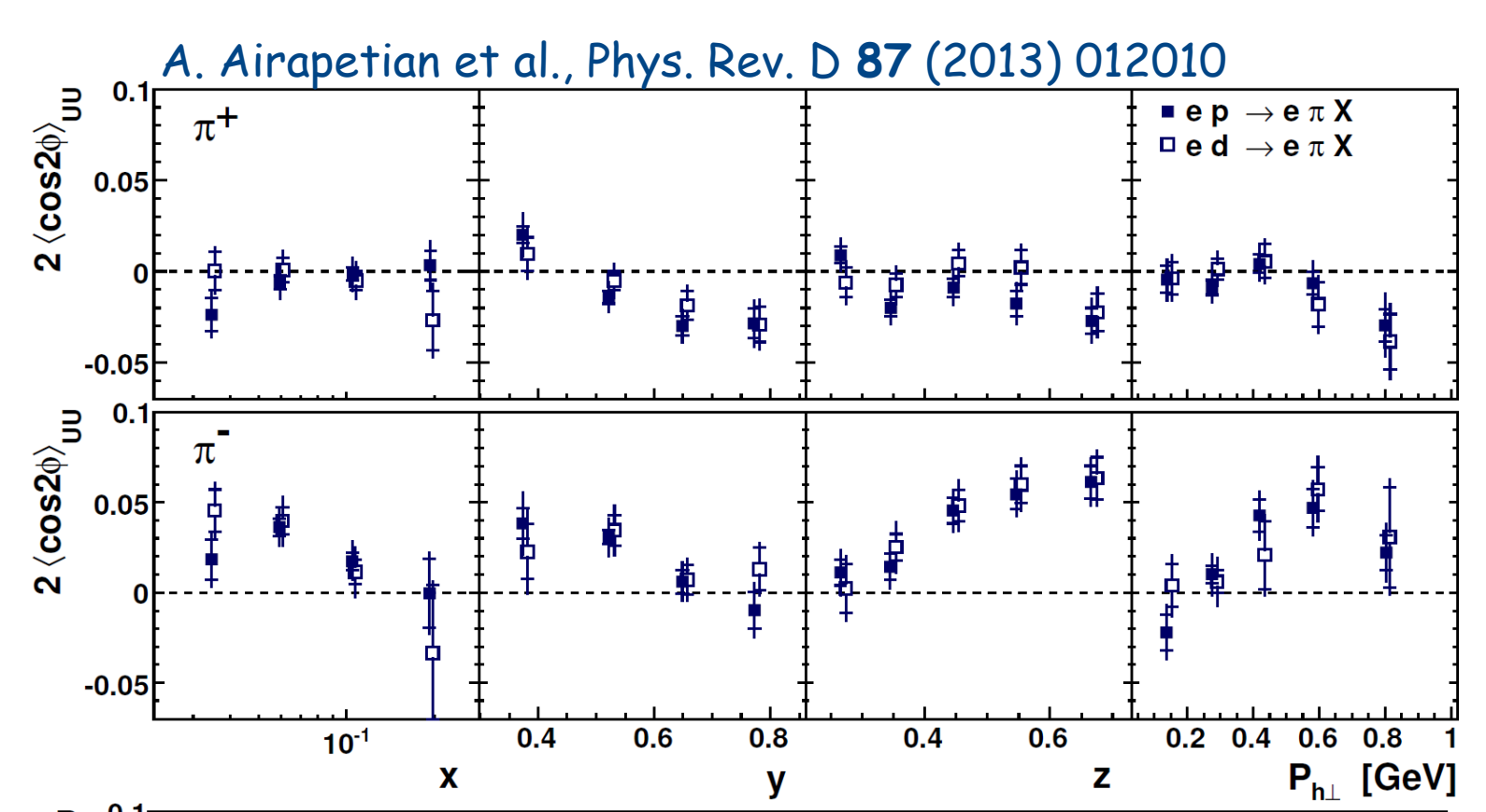

$$
F_{U U}^{\cos \left(2 \phi_{h}\right)} \propto h_{1}^{\perp} \otimes H_{1}^{\perp}
$$

- significant amplitudes orbital angular momentum

- H-D comparison: $h_{1}^{\perp, u} \approx h_{1}^{\perp, d}$

- $\pi^{-}>0 \leftrightarrow \pi^{+} \leqslant 0$, consistent with $H_{1}^{\perp, f a v} \approx-H_{1}^{\perp, u n f a v}$

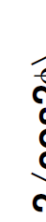

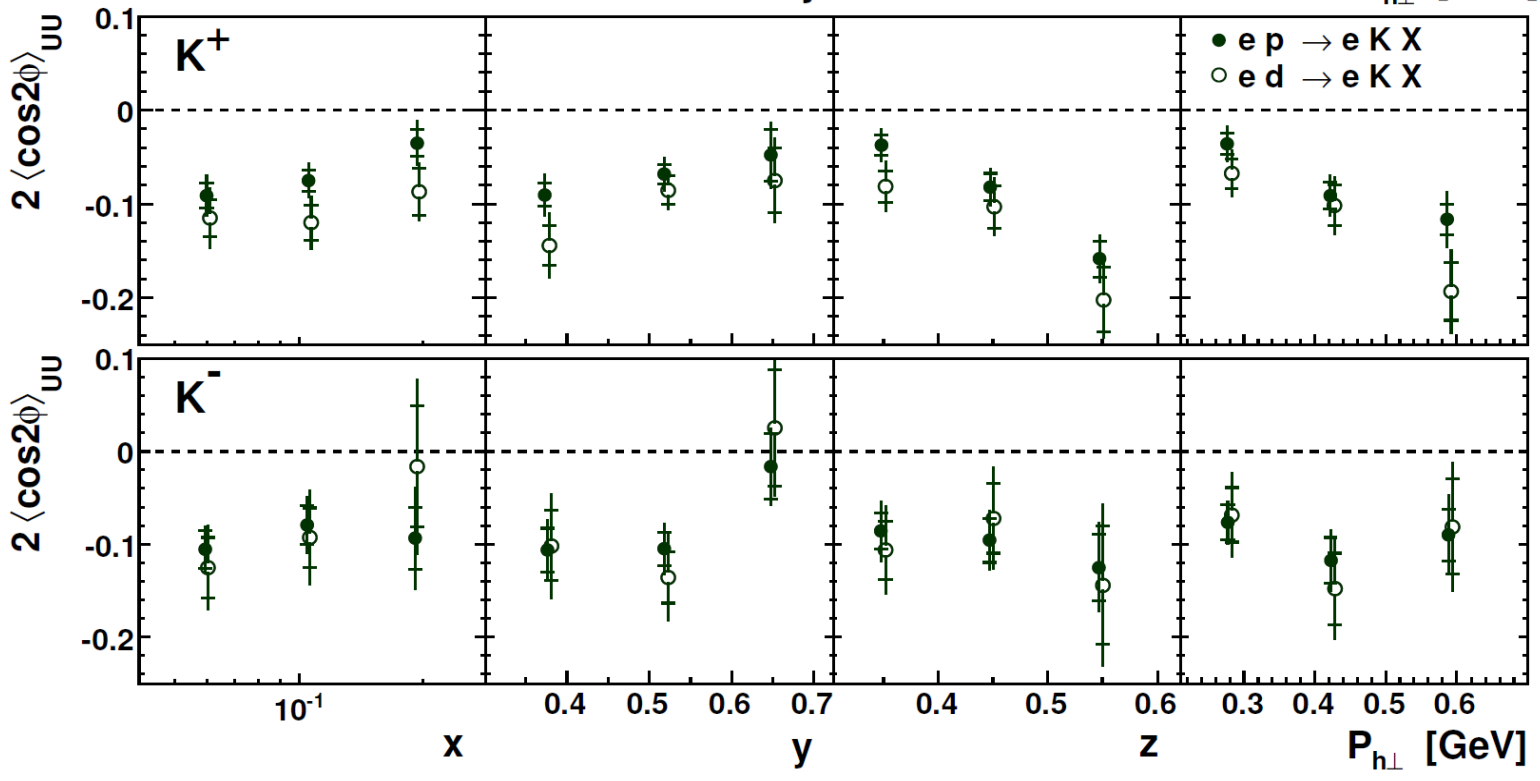

- $\mathrm{K}^{+} \& \mathrm{~K}^{-}$: large negative amplitudes

- $\mathrm{K}^{-} \simeq \mathrm{K}^{+}$

- $K^{ \pm}$very different from $\pi^{ \pm}$

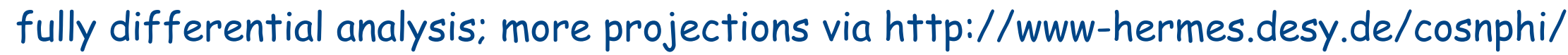




\section{Twist-3: Cahn, Boer-Mulders, ...}

A. Airapetian et al., Phys. Rev. D 87 (2013) 012010

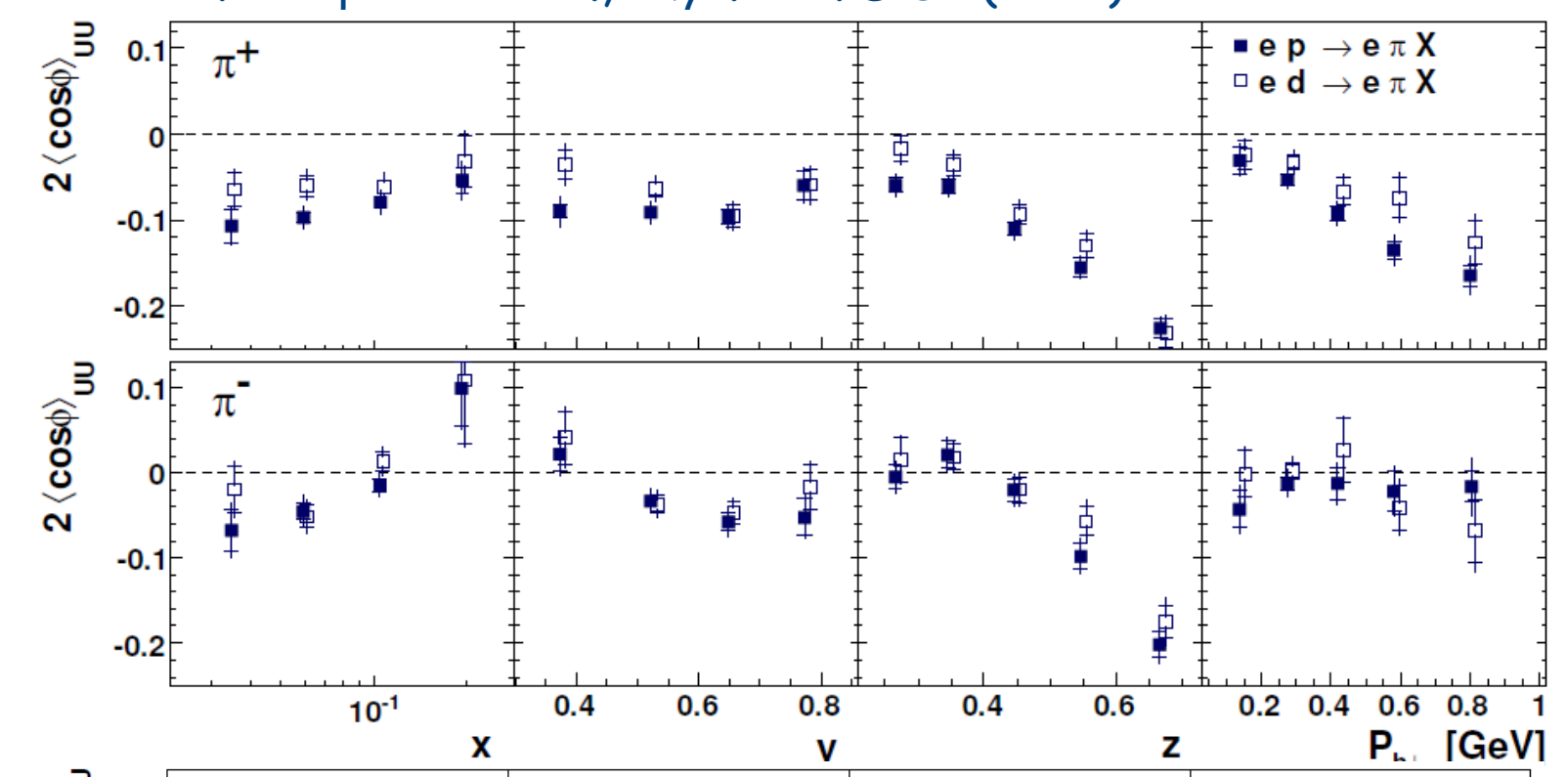

$$
F_{U U}^{\cos \phi_{h}} \propto \frac{M}{Q}\left[f_{1} \times D_{1}, h_{1}^{\perp} \times H_{1}^{\perp}, \ldots\right]
$$

- H-D comparison:

weak flavour dependence

- strong flavour dependence

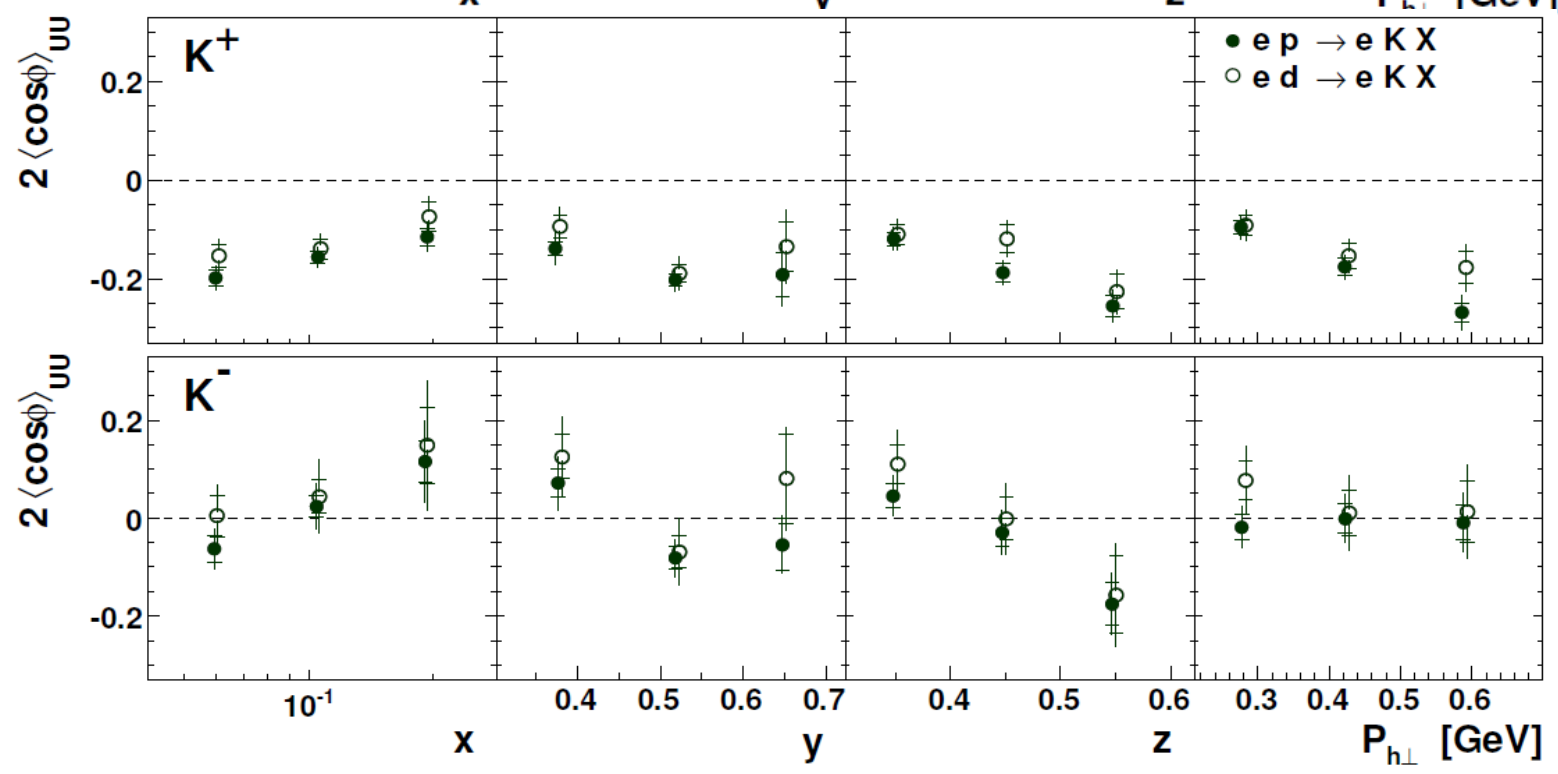

fully differential analysis; more projections via http://www-hermes.desy.de/cosnphi/ 
DIS with transversely polarized target 


\section{Structure function $g_{2}$}

- $\frac{d^{3} \sigma_{L T}}{d x d y d \phi^{\prime}} \propto-h_{k}\left(\frac{y}{2} g_{1}\left(x, Q^{2}\right)+g_{2}\left(x, Q^{2}\right)\right) \cos \left(\phi^{\prime}\right)$

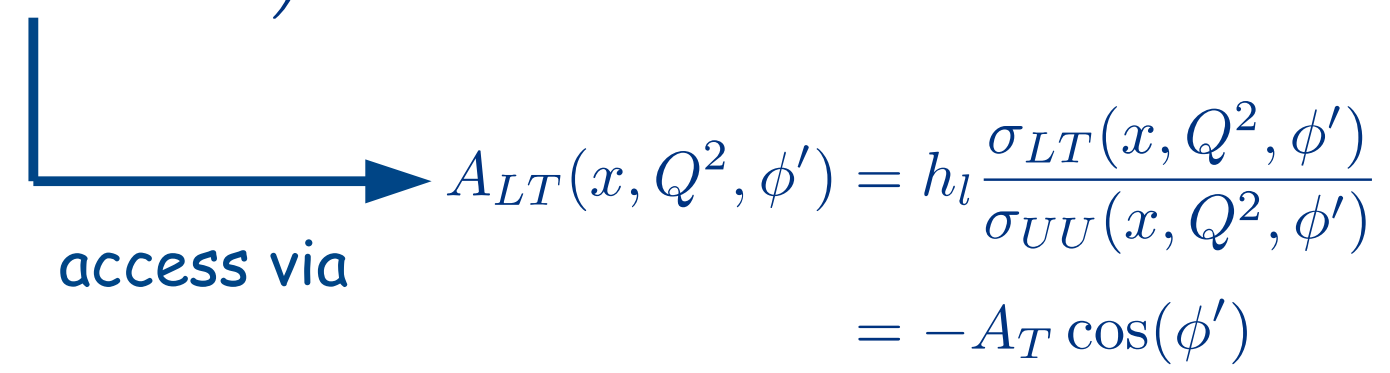

- $g_{2}(x)=g_{2}^{W W}+\bar{g}_{2}(x) \quad d_{2}=3 \int_{0}^{1} d x x^{2} \bar{g}_{2}(x)$ 


\section{Structure function $g_{2}$}

- $\frac{d^{3} \sigma_{L T}}{d x d y d \phi^{\prime}} \propto-h_{k}\left(\frac{y}{2} g_{1}\left(x, Q^{2}\right)+g_{2}\left(x, Q^{2}\right)\right) \cos \left(\phi^{\prime}\right)$

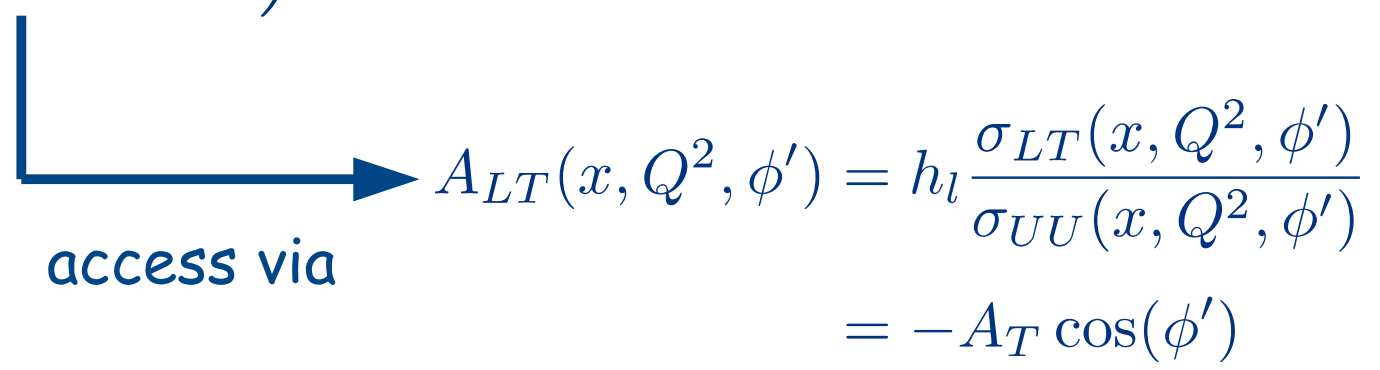

- $g_{2}(x)=g_{2}^{W W}+\bar{g}_{2}(x) \quad d_{2}=3 \int_{0}^{1} d x x^{2} \bar{g}_{2}(x)$

- $\quad$ Sivers effect

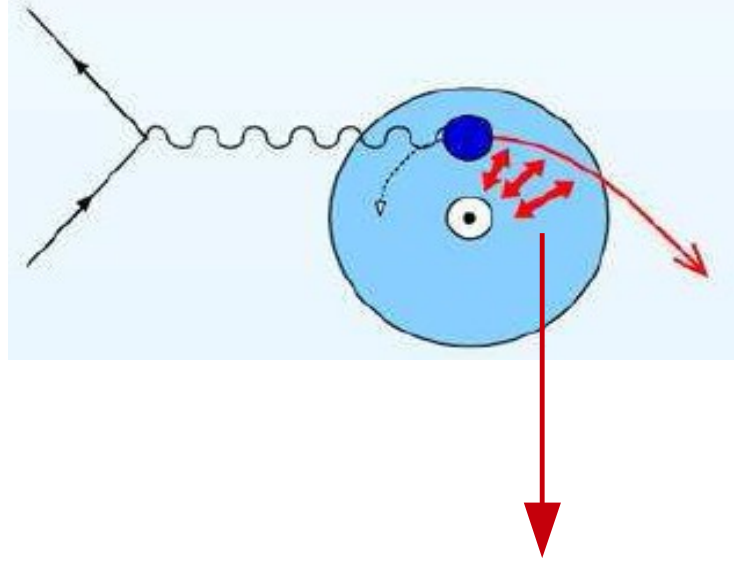

force on struck quark at $t=0$

$\propto-d_{2}$

M. Burkardt, arXiv:0810.3589

FSI: $\dagger=0 \rightarrow \infty$ 

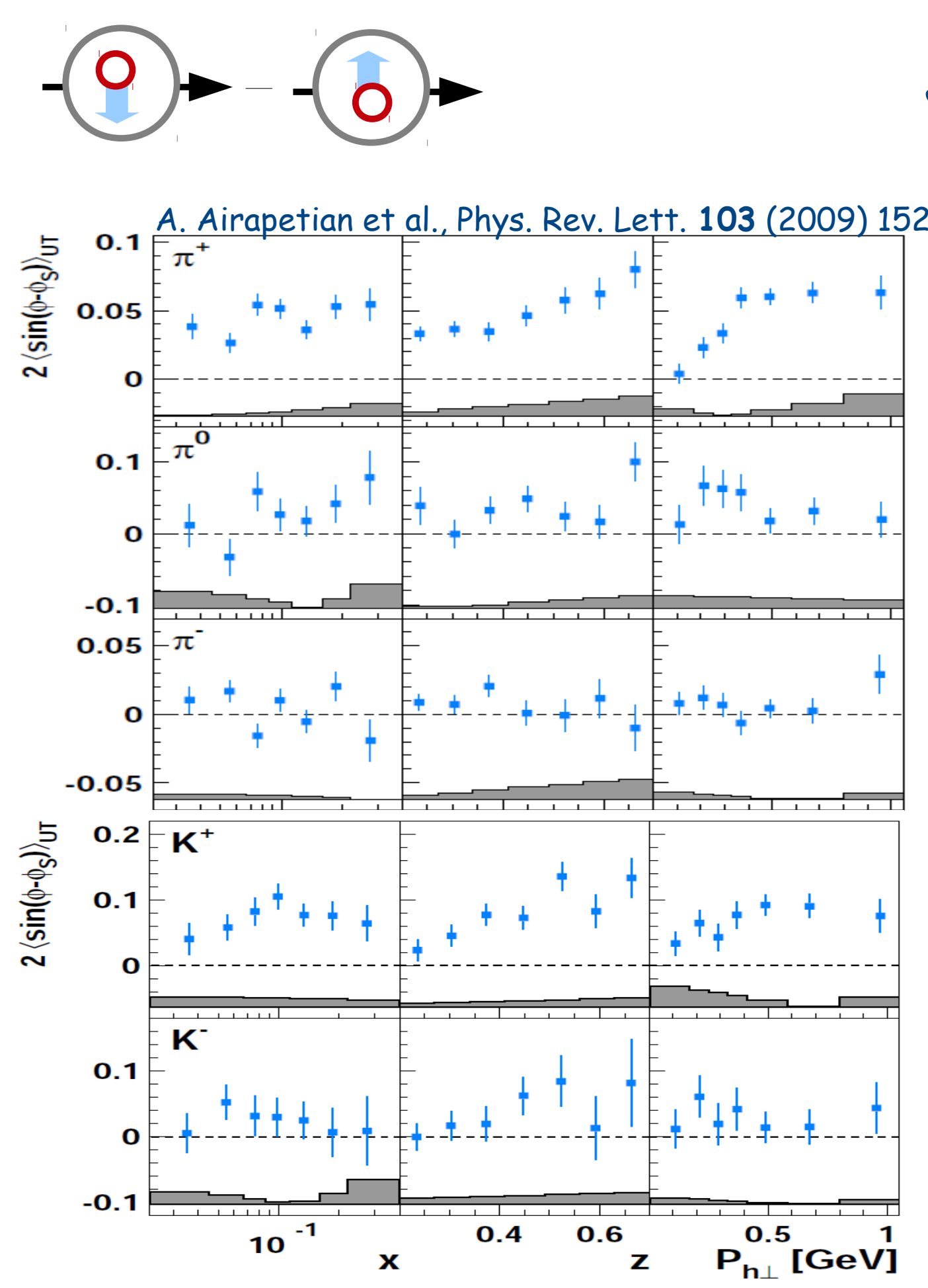

Sivers function $f_{1 T}^{\perp}$

$$
F_{U T}^{\sin \left(\phi_{h}-\phi_{S}\right)} \propto f_{1 T}^{\perp} \otimes D_{1}
$$

- $\pi^{+}$: significantly positive

$$
\text { orbital angular momentum }
$$

- u-quark dominance for $\mathrm{p}^{+}$amplitude:

$$
\begin{gathered}
\approx-\frac{f_{1 T}^{\perp, u}\left(x, k_{T}^{2}\right) \otimes D_{1}^{u \rightarrow \pi^{+}}\left(z, p_{T}^{2}\right)}{f_{1}^{u}\left(x, k_{T}^{2}\right) \otimes D_{1}^{u \rightarrow \pi^{+}}\left(z, p_{T}^{2}\right)} \\
\square \quad f_{1 T}^{\perp, u}\left(x, k_{T}^{2}\right)<0
\end{gathered}
$$

- $\pi^{-}: u$ - and d-quark cancellation

$$
f_{1 T}^{\perp, d}\left(x, k_{T}^{2}\right)>0
$$




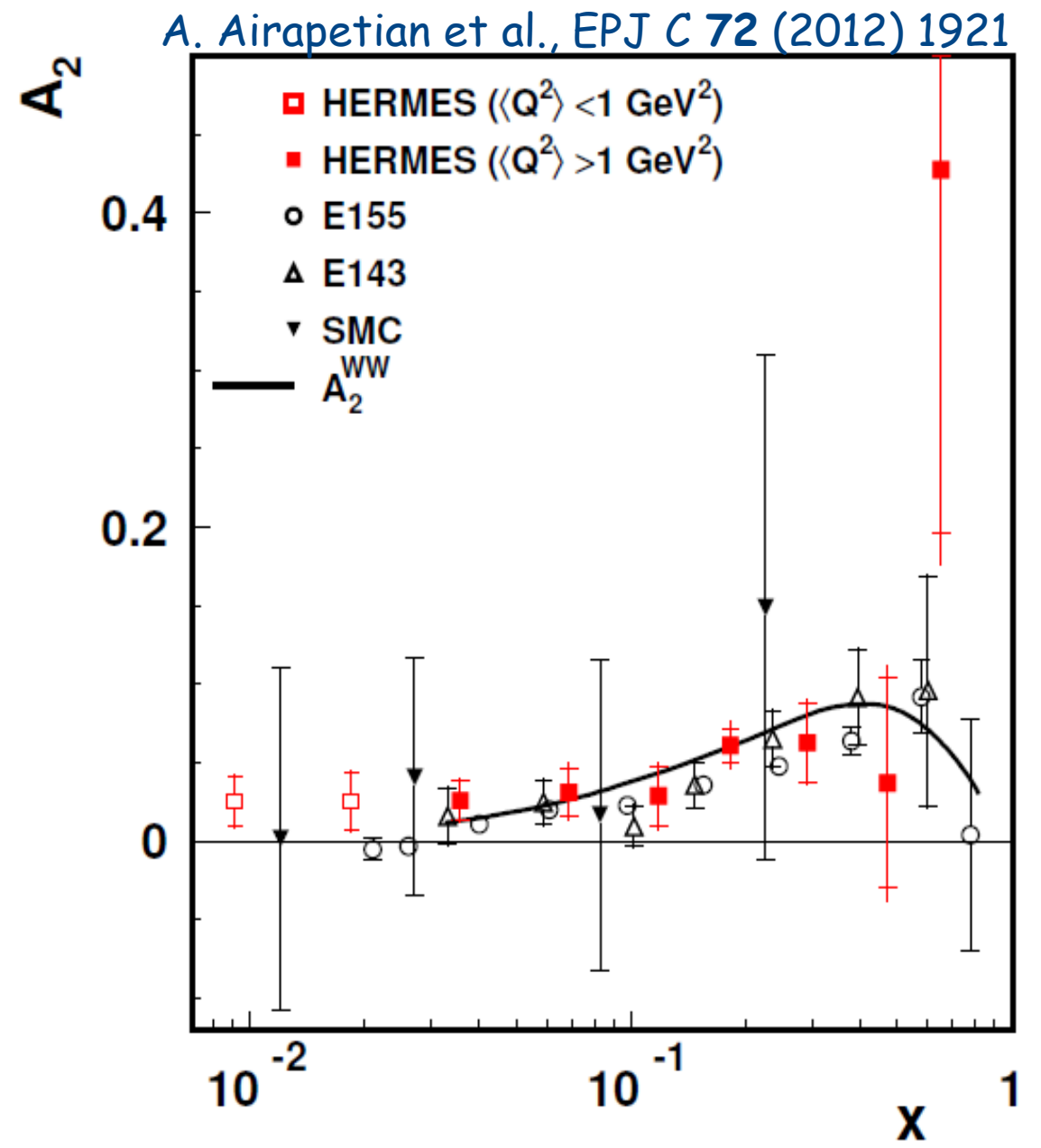

- evaluation of Burkhardt-Cottingham sum rule:

$$
\int_{0.023}^{0.9} d x g_{2}(x)=0.006 \pm 0.024 \pm 0.017
$$

$\mathrm{E} 143+\mathrm{E} 155: \int_{0.02}^{0.8} d x g_{2}(x)=-0.042 \pm 0.008$

\section{$A_{2}$ and $g_{2}$}

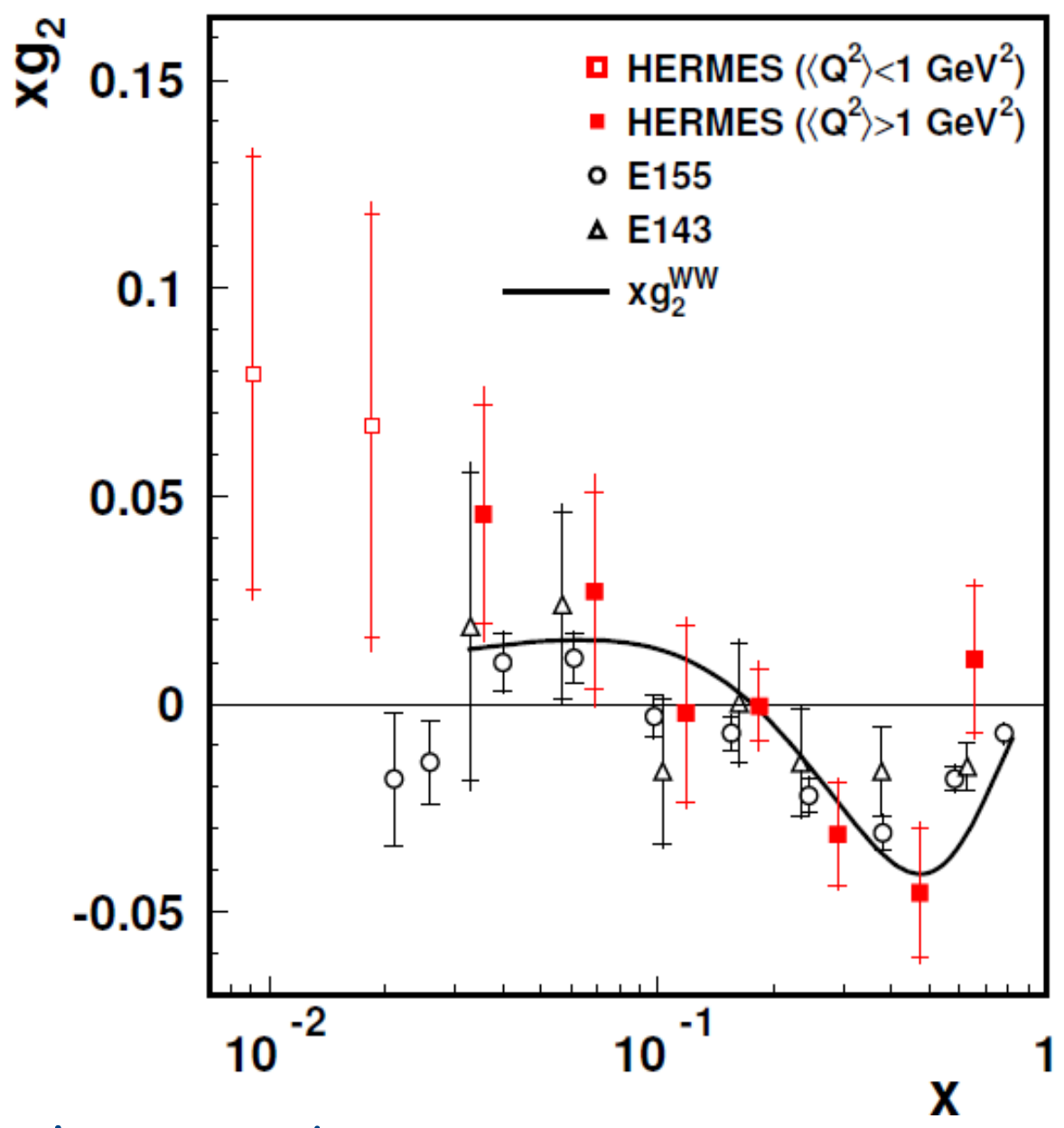

- $\mathrm{d}_{2}$ :

$d_{2}=0.0148 \pm 0.0096 \pm 0.0048$

E143+E155:

$d_{2}=0.0032 \pm 0.0017$ 


\section{The nucleon in multiple dimensions}

Wigner distributions $W\left(x, \vec{k}_{T}, \overrightarrow{b_{\perp}}\right)$

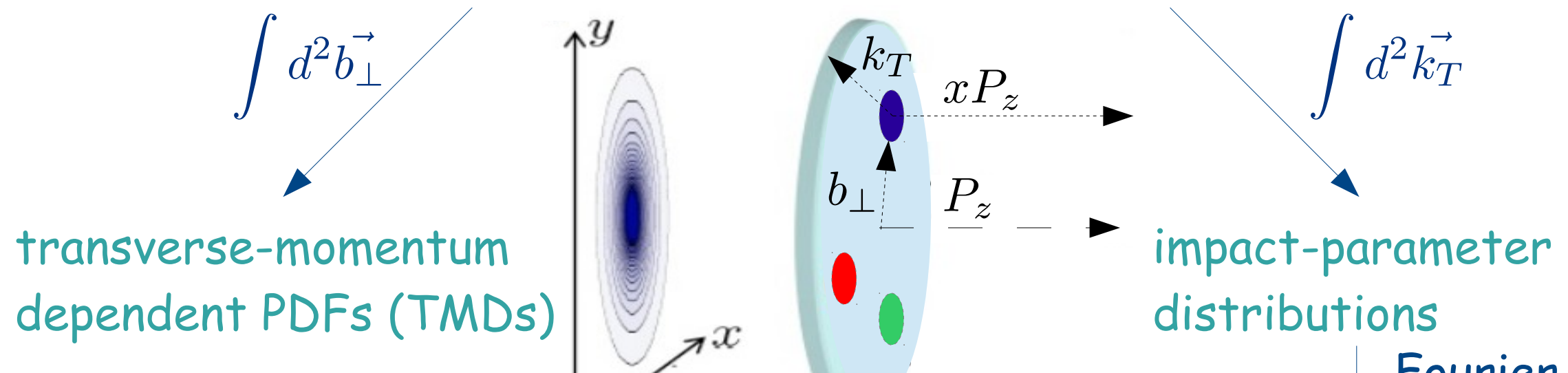

semi-inclusive deep-inelastic scattering (DIS)

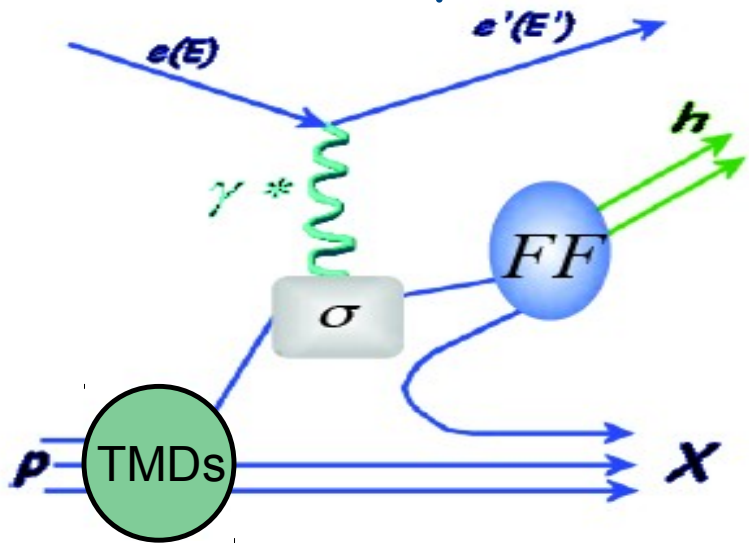

Fourier

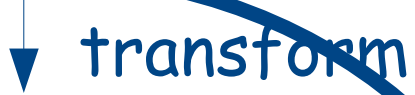

generalized parton distributions (GPDs) hard exclusive reactions

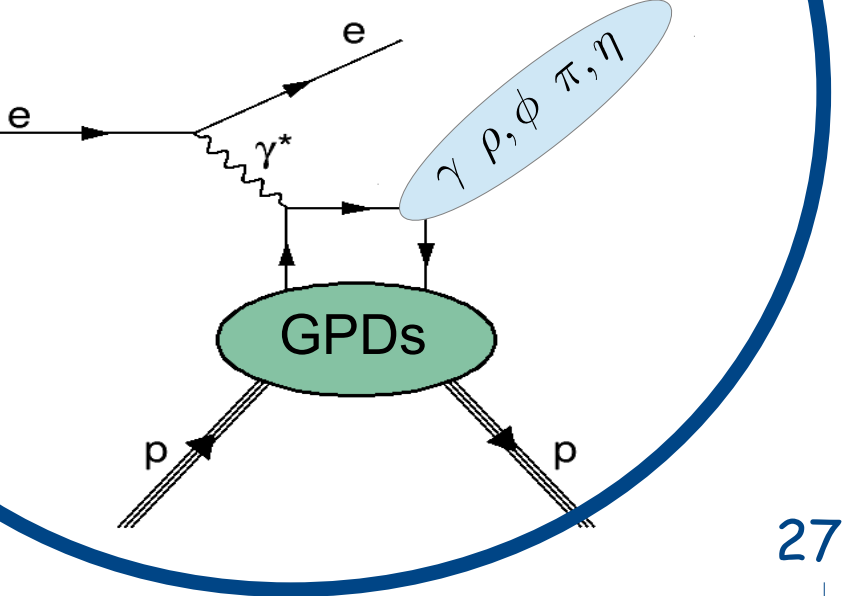




\section{Deeply virtual Compton scattering}




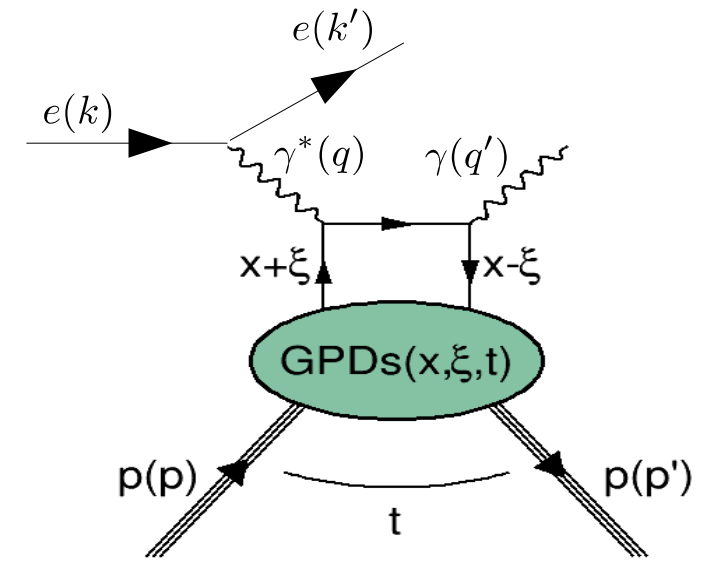

DVCS

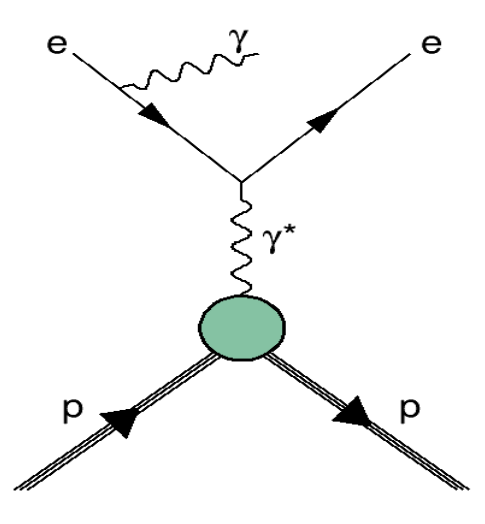

Bethe-Heitler

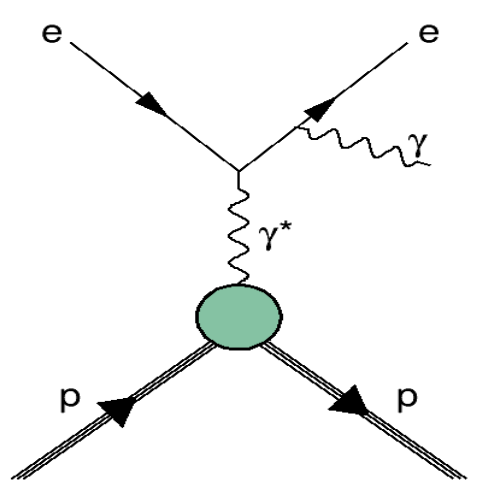

$C S \tau_{B H}^{*}$

$d \sigma \propto\left|\tau_{B H}\right|^{2}+\left|\tau_{D V C S}\right|^{2}+\tau_{B H} \tau_{D V C S}^{*}+\tau_{D V C S}$
$\longrightarrow$ access through azimuthal asymmetries

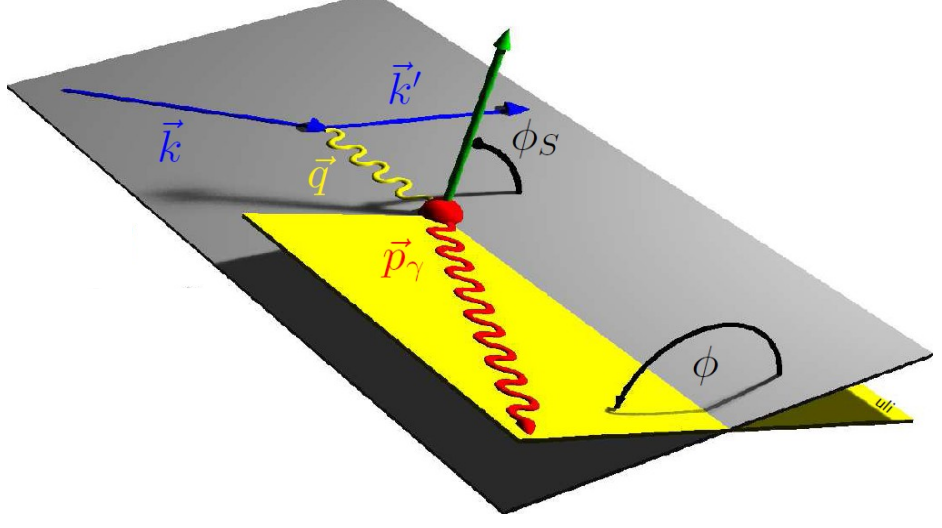




\section{Exclusive electroproduction of}

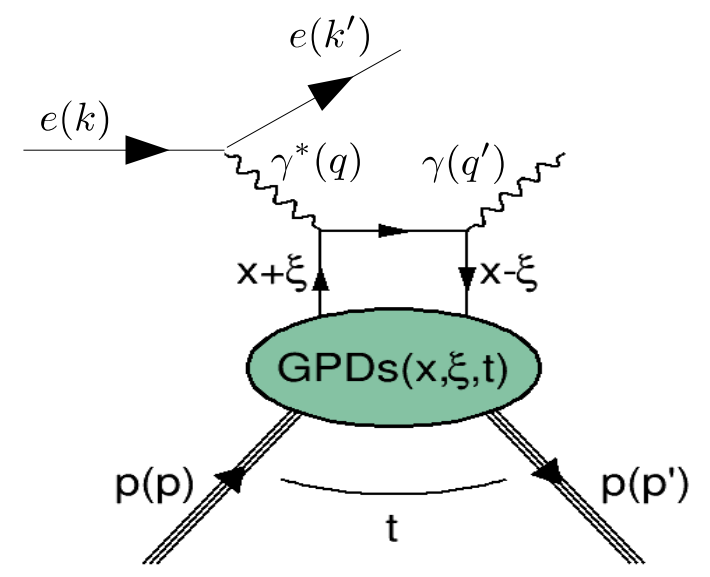

DVCS

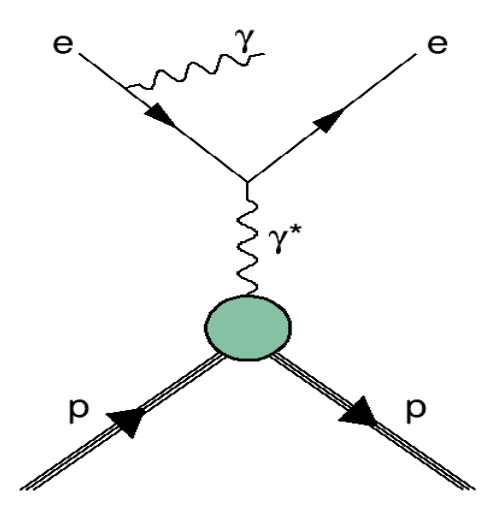

Bethe-Heitler

$d \sigma \propto\left|\tau_{B H}\right|^{2}+\left|\tau_{D V C S}\right|^{2}+\tau_{B H} \tau_{D V C S}^{*}+\tau_{D V C S} \tau_{B H}^{*}$

- access through azimuthal asymmetries

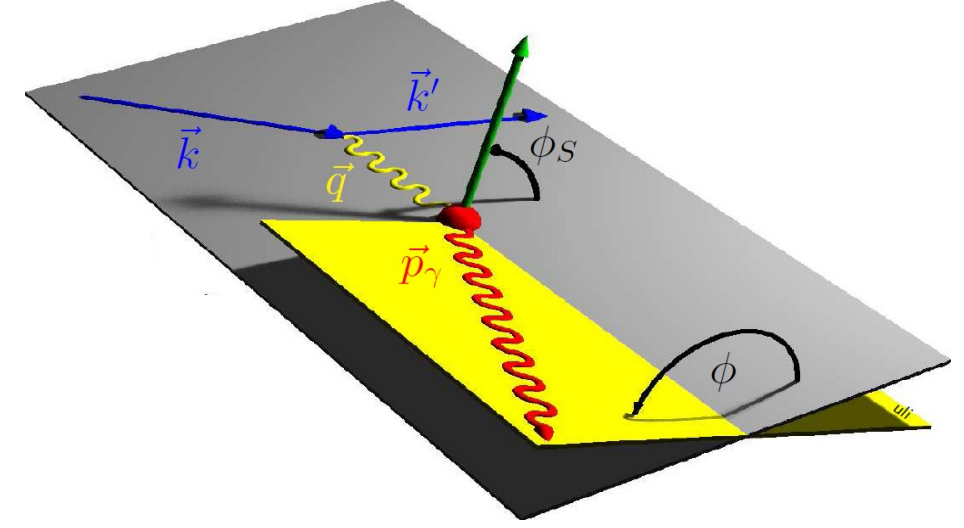

real photons

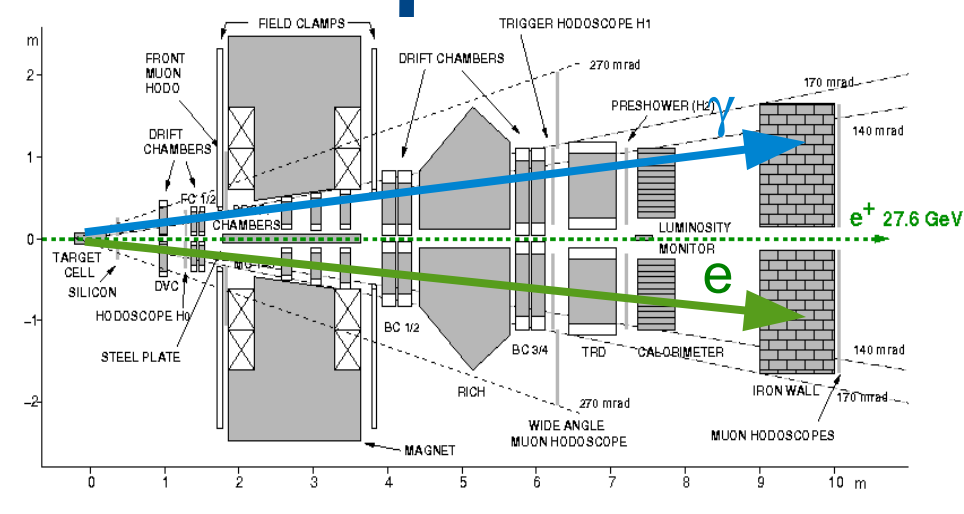

\section{exclusivity}

reconstruct proton via missing mass:

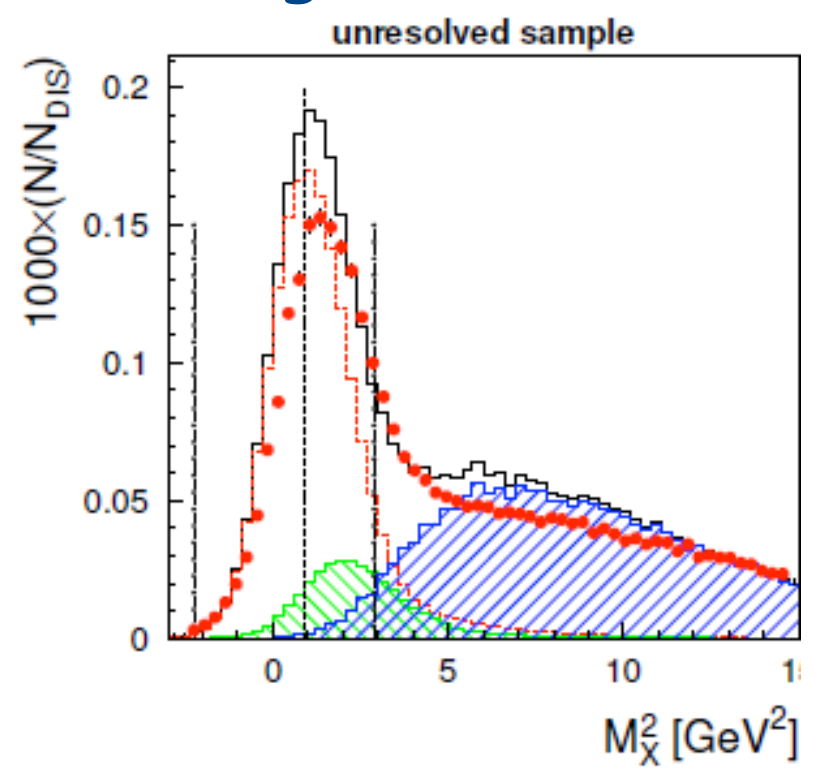




\section{Double spin asymmetries on}

GPD $E$

transversely polarized target

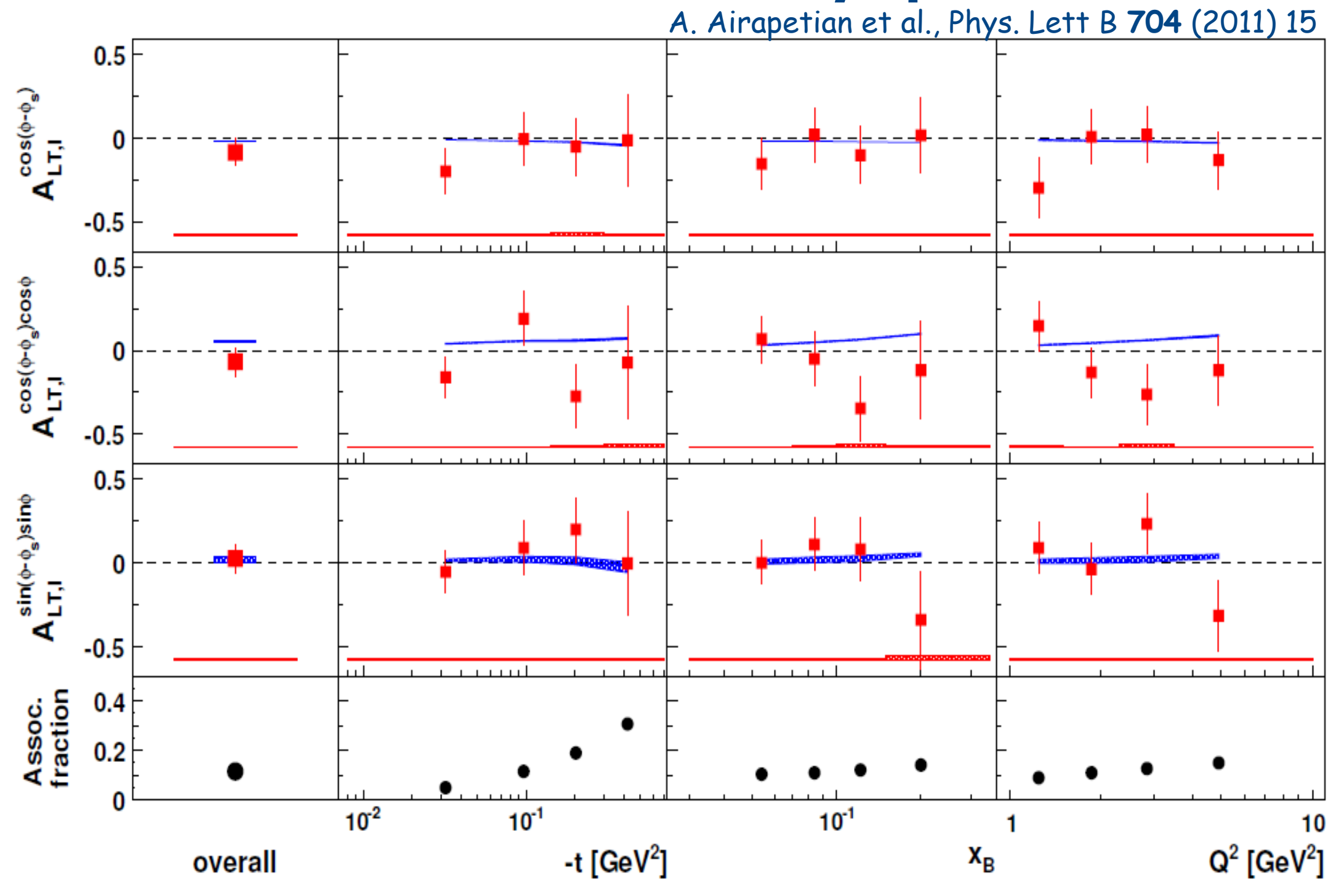

phys. Rev D 60 (1999) 094017 + VGG code:

- double distributions

- Regge t-dependence

$-0.2<J u<0.6 \& J d=0$

- compatible with zero 


\section{Charged-separated beam-helicity}

GPD $H$

asymmetry

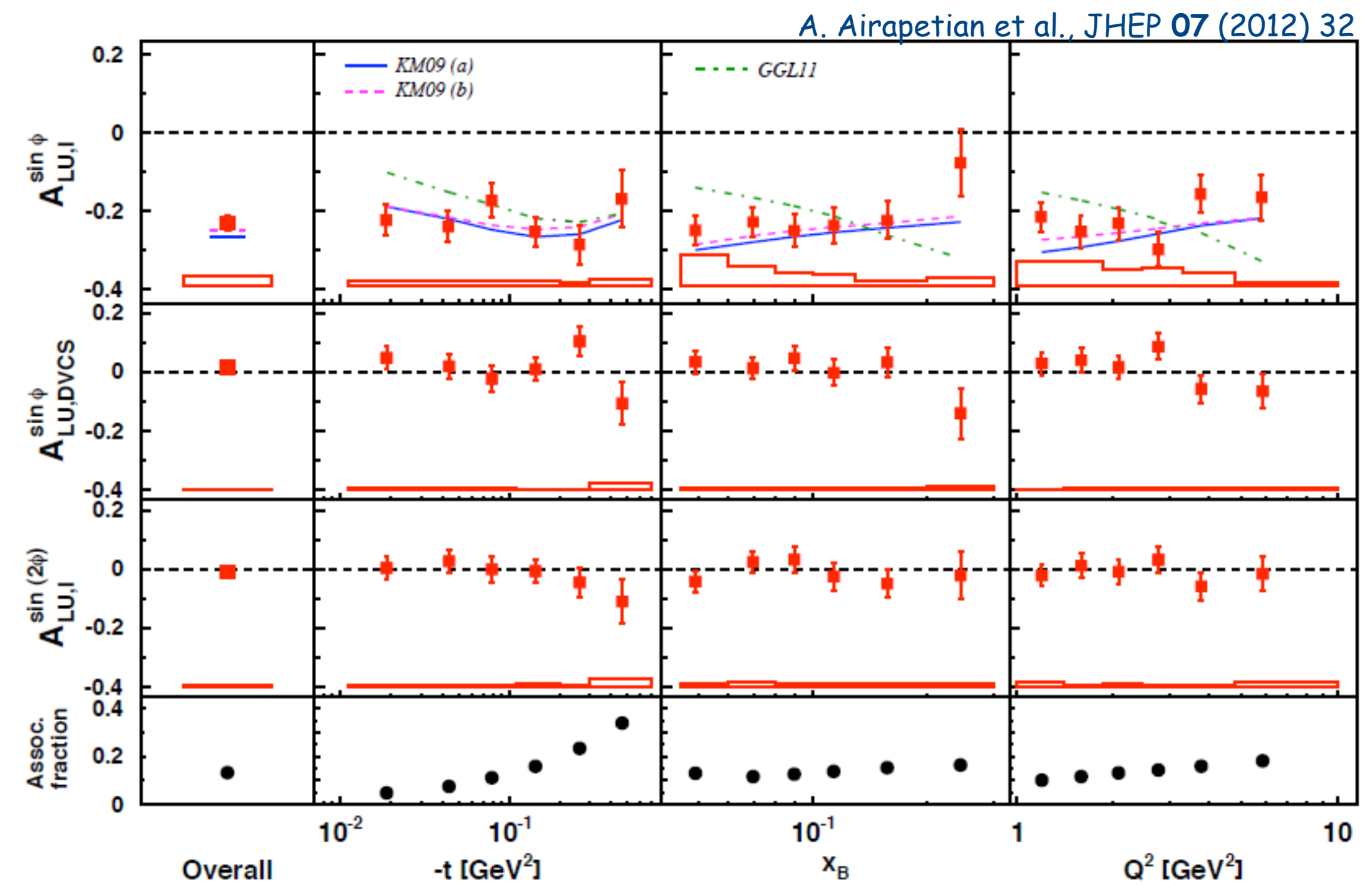

KM09: Nucl. Phys. B 841 (2010) 1:

fit to HERMES, ZEUS, H1 data

Fit to HERMES, ZEUS,

H1, Jefferson Lab data

GGL11:Phys. Rev. D

84 (2011) 034007

- data collected from 1996-2007 (74\% of data from 2006-2007)

- additional $3.2 \%$ scale uncertainty from beam polarization 


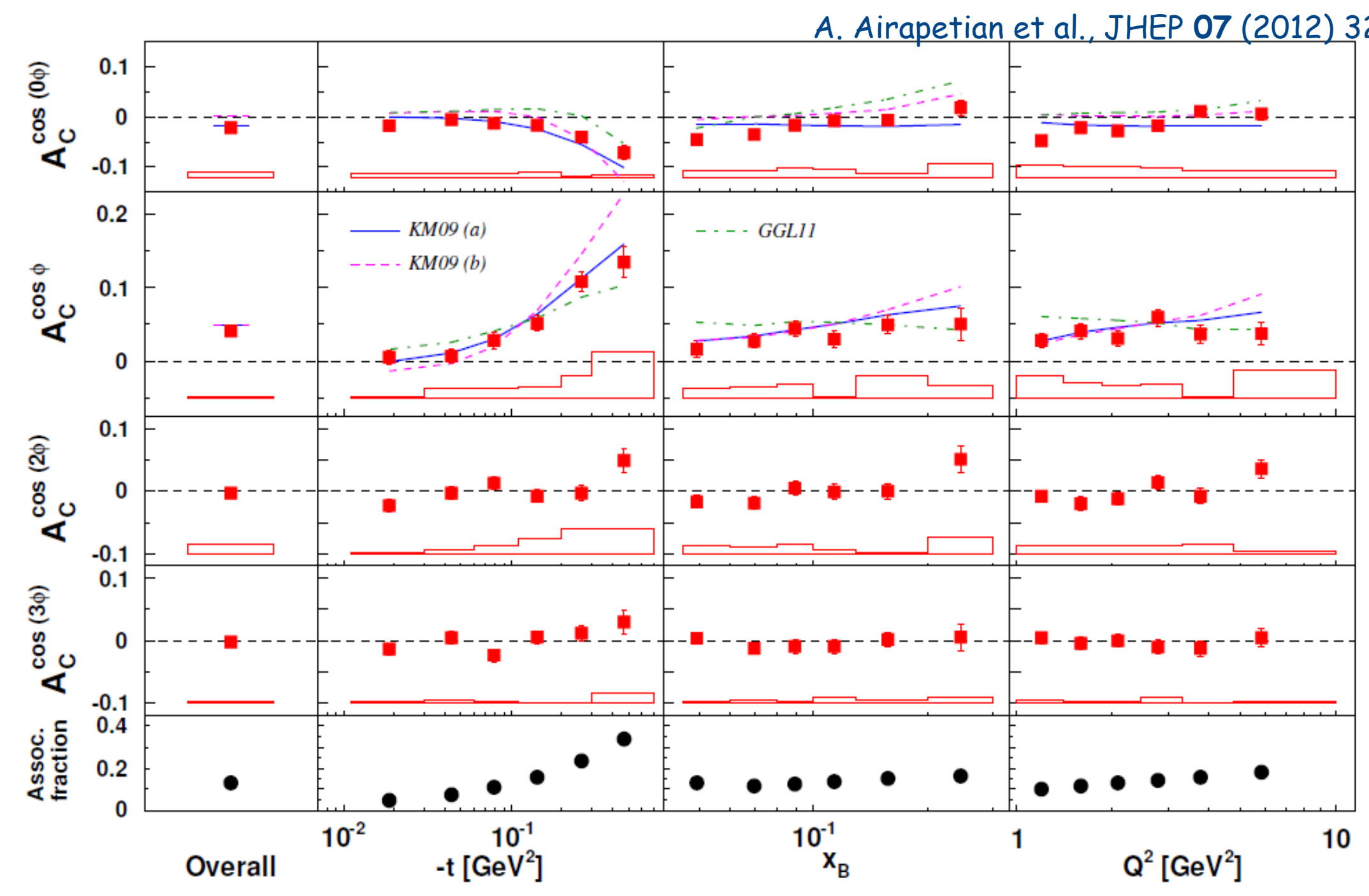

KM09: Nucl. Phys. B 841 (2010) 1:

fit to HERMES, ZEUS, H1 data

Fit to HERMES, ZEUS, H1, Jefferson Lab data

GGL11:Phys. Rev. D 84 (2011) 034007

- data collected from 1996-2007 (74\% of data from 2006-2007)

- additional $3.2 \%$ scale uncertainty from beam polarization 


\section{DVCS at HERMES}

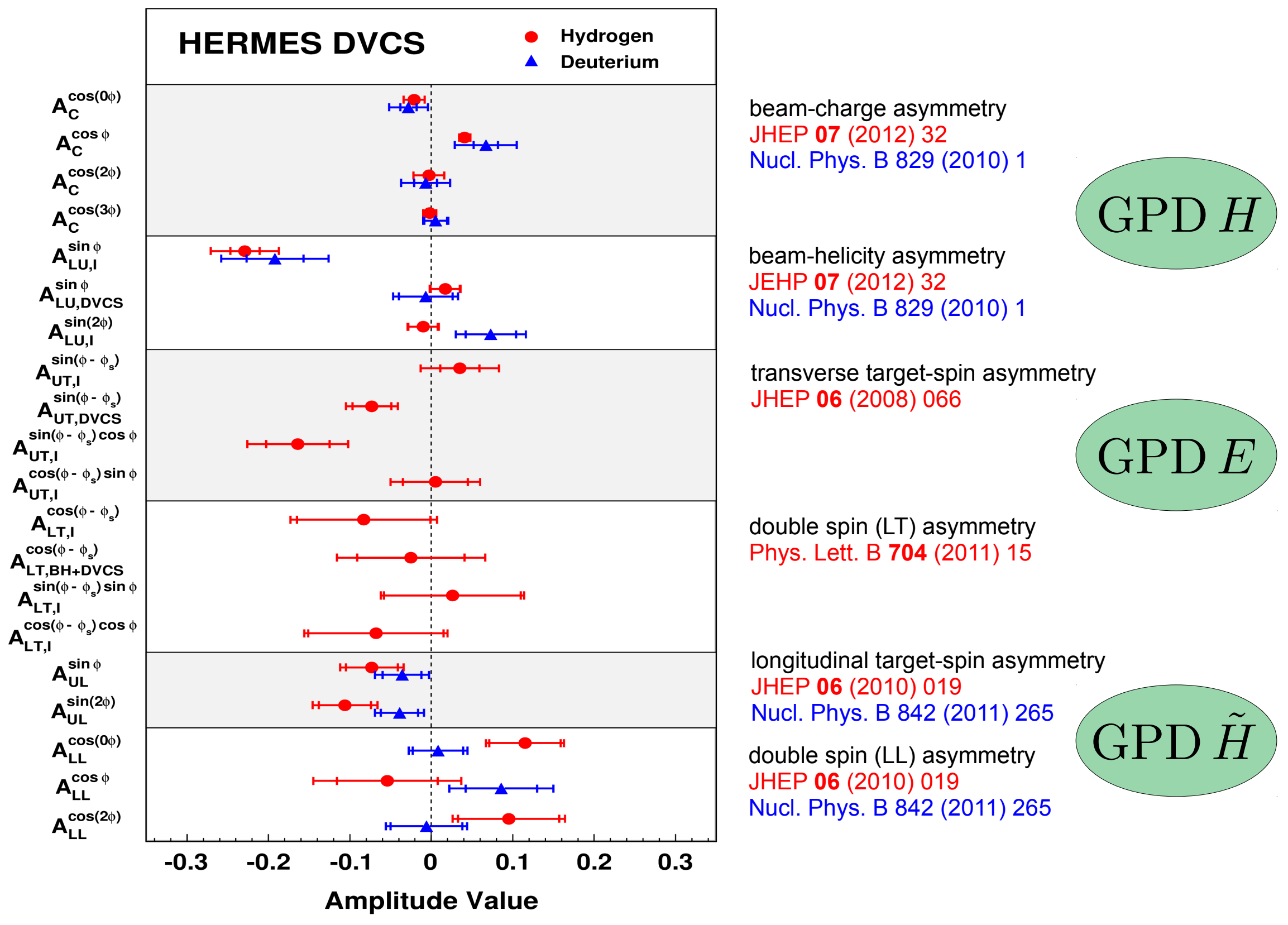




\section{DVCS/BH complete event reconstruction}
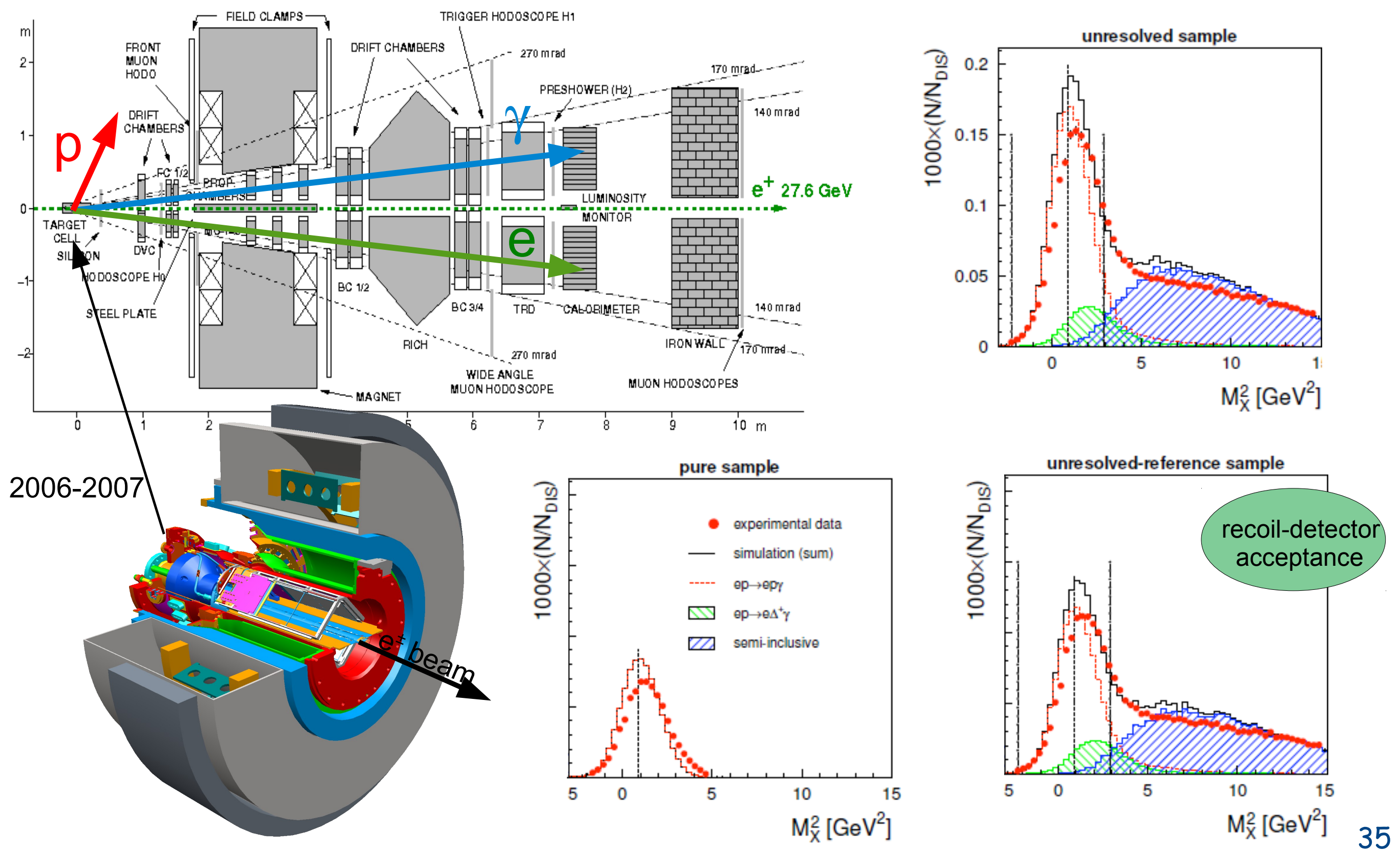


\section{Beam-helicity asymmetry}

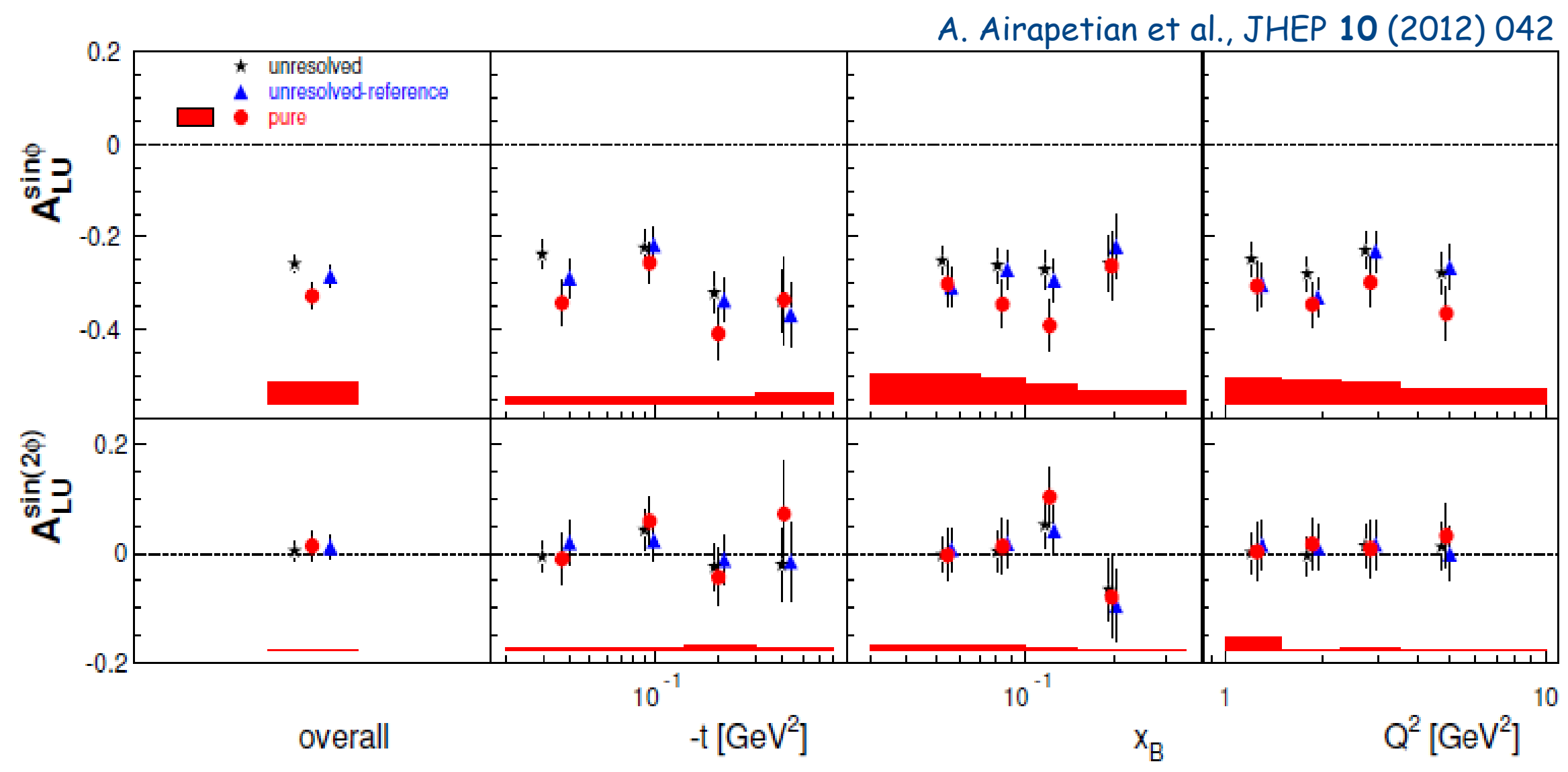

- additional $1.96 \%$ scale uncertainty from beam polarization

- leading asymmetry from pure sample is larger 


\section{Beam-helicity asymmetry in}

GPDs

\section{$\mathrm{ep} \rightarrow \mathrm{e} \gamma \pi \mathrm{N}$ in $\Delta$-resonance region}

quark distribution in longitudinal-momentum and transverse-position space
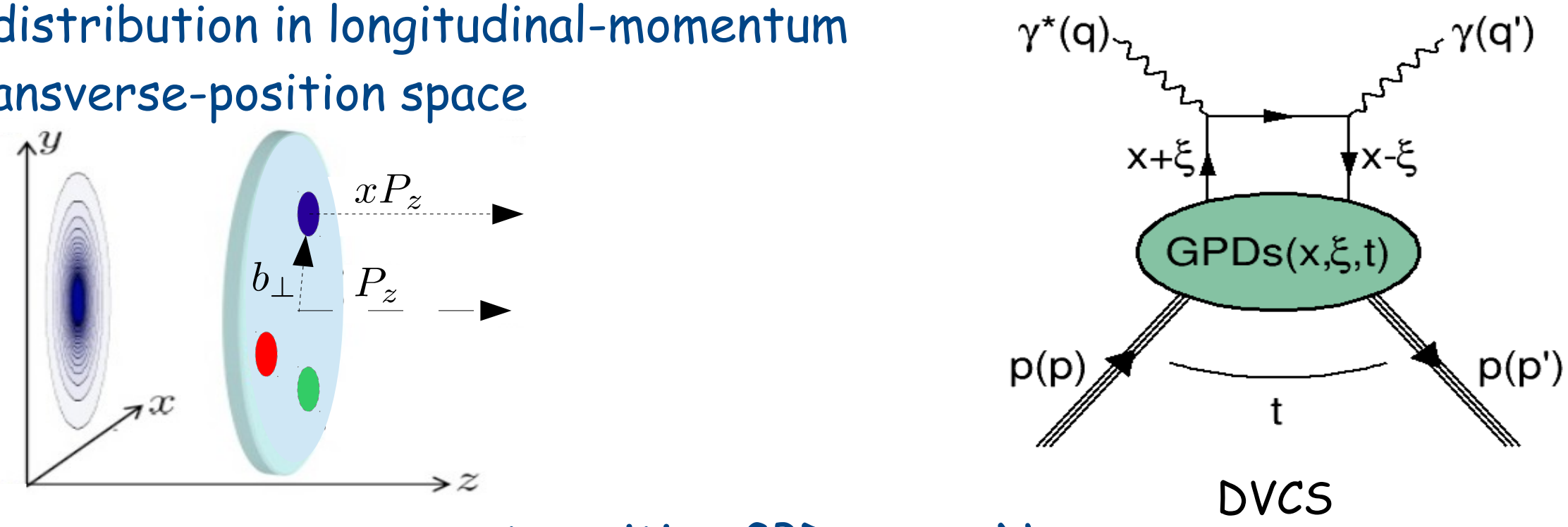

transition GPDs $p \rightarrow \pi \mathrm{N}$

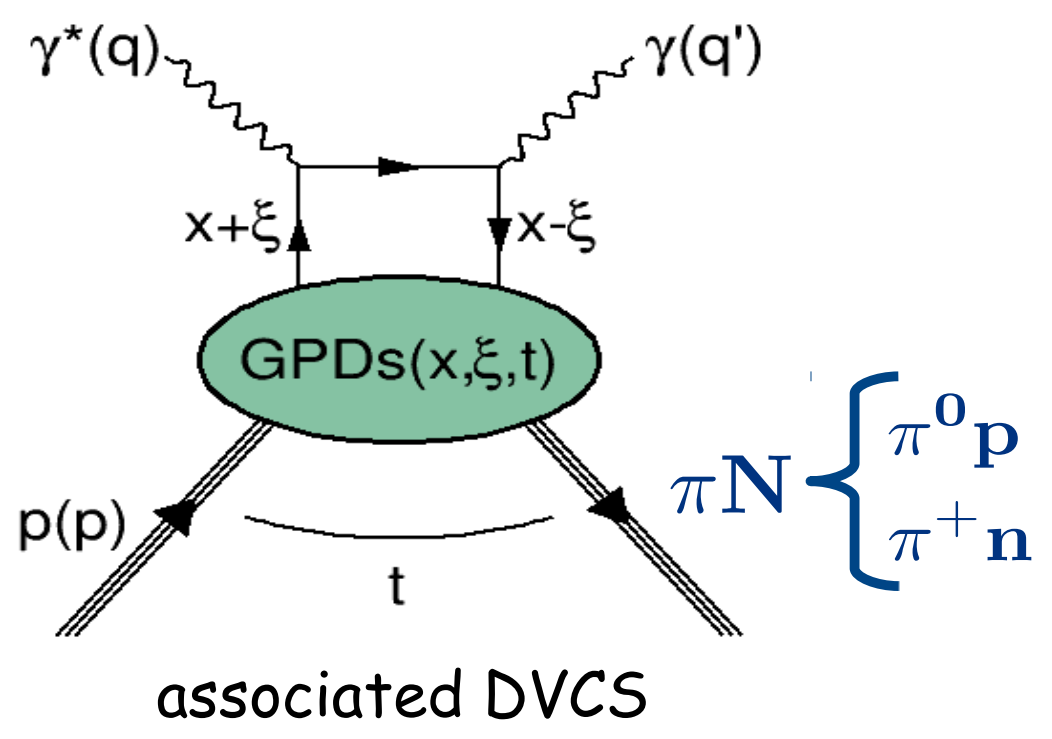




\section{Beam-helicity asymmetry in}

\section{$\mathrm{ep} \rightarrow \mathrm{e} \gamma \pi \mathrm{N}$ in $\Delta$-resonance region}

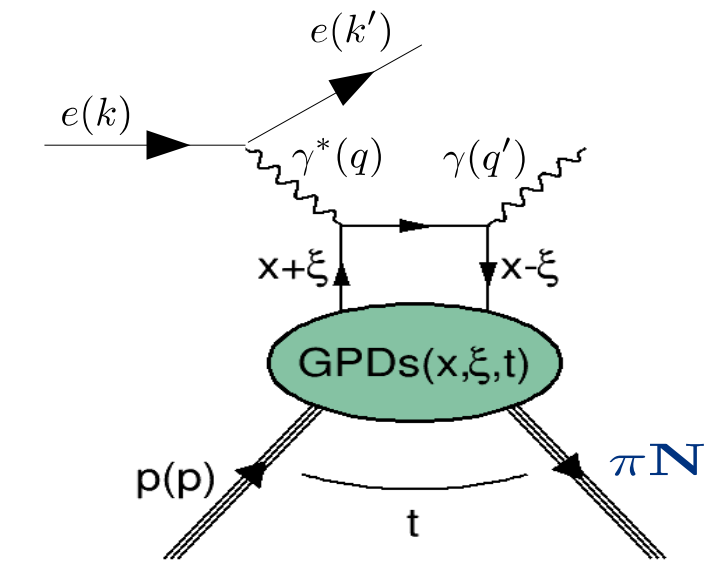

associated DVCS

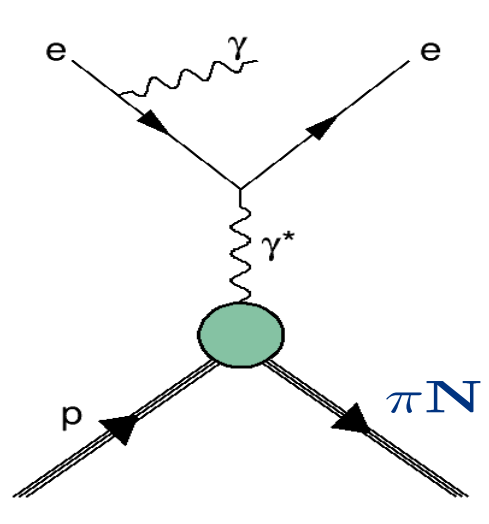

associated Bethe-Heitler $d \sigma \propto\left|\tau_{B H}\right|^{2}+\left|\tau_{D V C S}\right|^{2}+\tau_{B H} \tau_{D V C S}^{*}+\tau_{D V C S} \tau_{B H}^{*}$

- access through azimuthal asymmetries

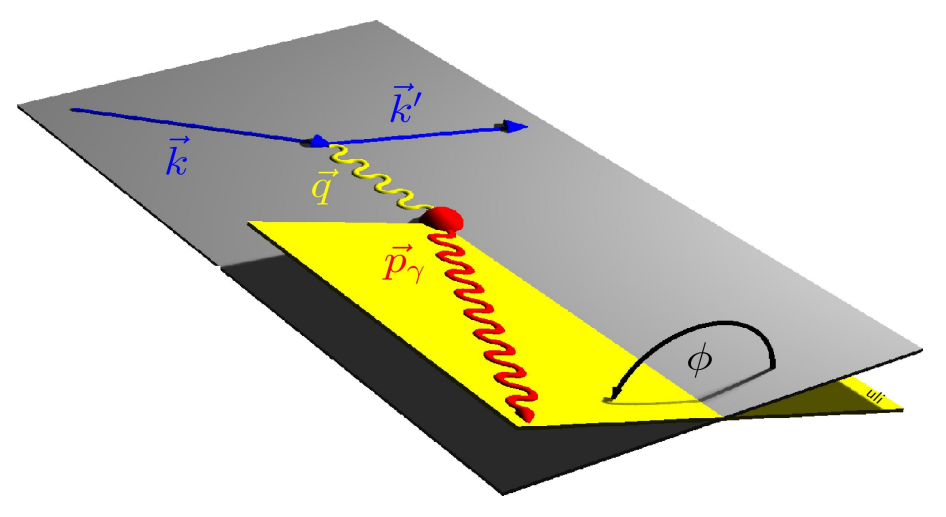




\section{Beam-helicity asymmetry in}

$\mathrm{ep} \rightarrow \mathrm{e} \gamma \pi^{0} \mathrm{p}$ in $\Delta$-resonance region

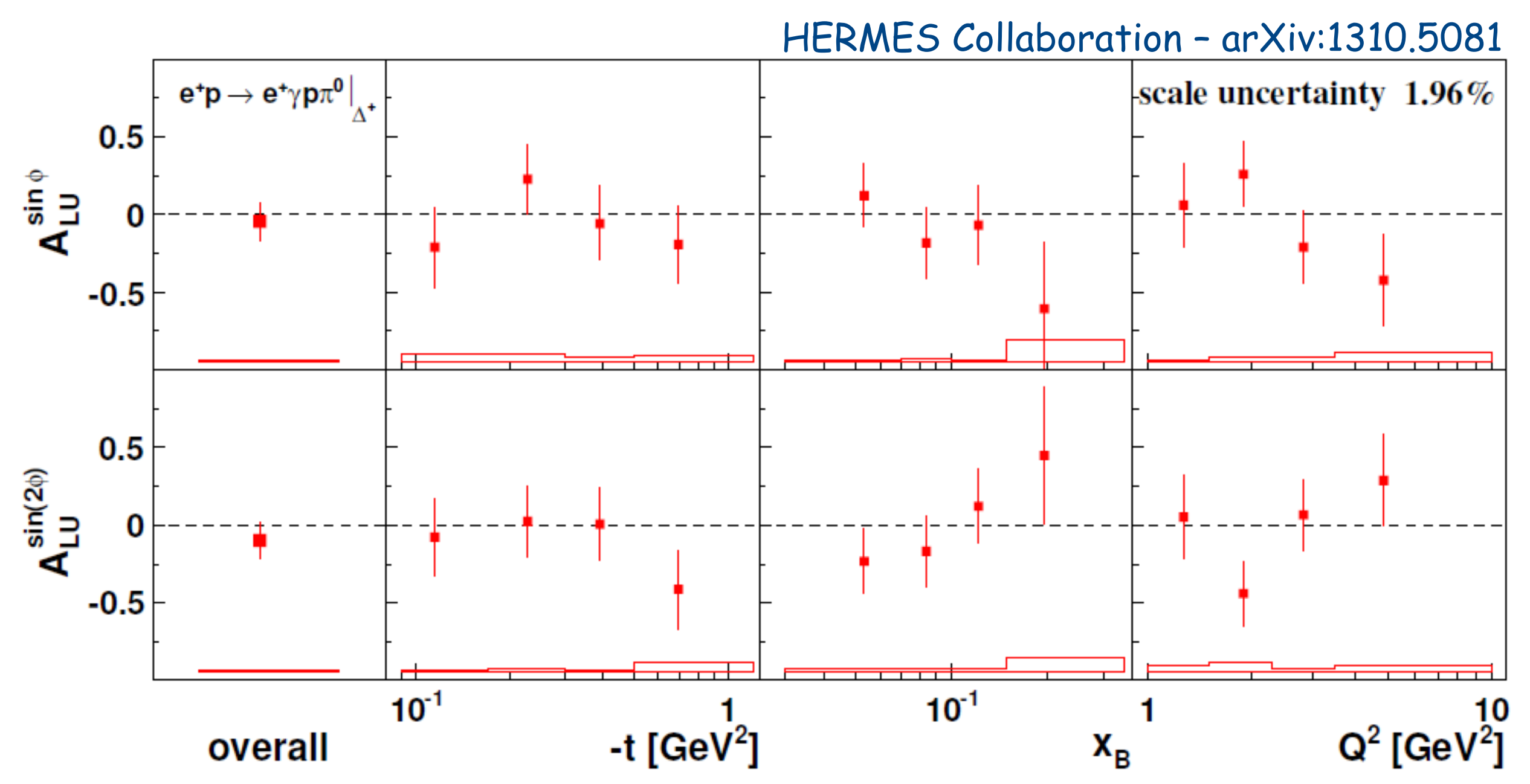

- asymmetry background correction from SIDIS (11\%) and ep $\rightarrow$ e $\gamma \mathbf{p}$ (4.6\%)

- leading asymmetry consistent with zero 


\section{Beam-helicity asymmetry in}

\section{$\mathbf{e p} \rightarrow \mathbf{e} \gamma \pi^{+} \mathbf{n}$ in $\Delta$-resonance region}

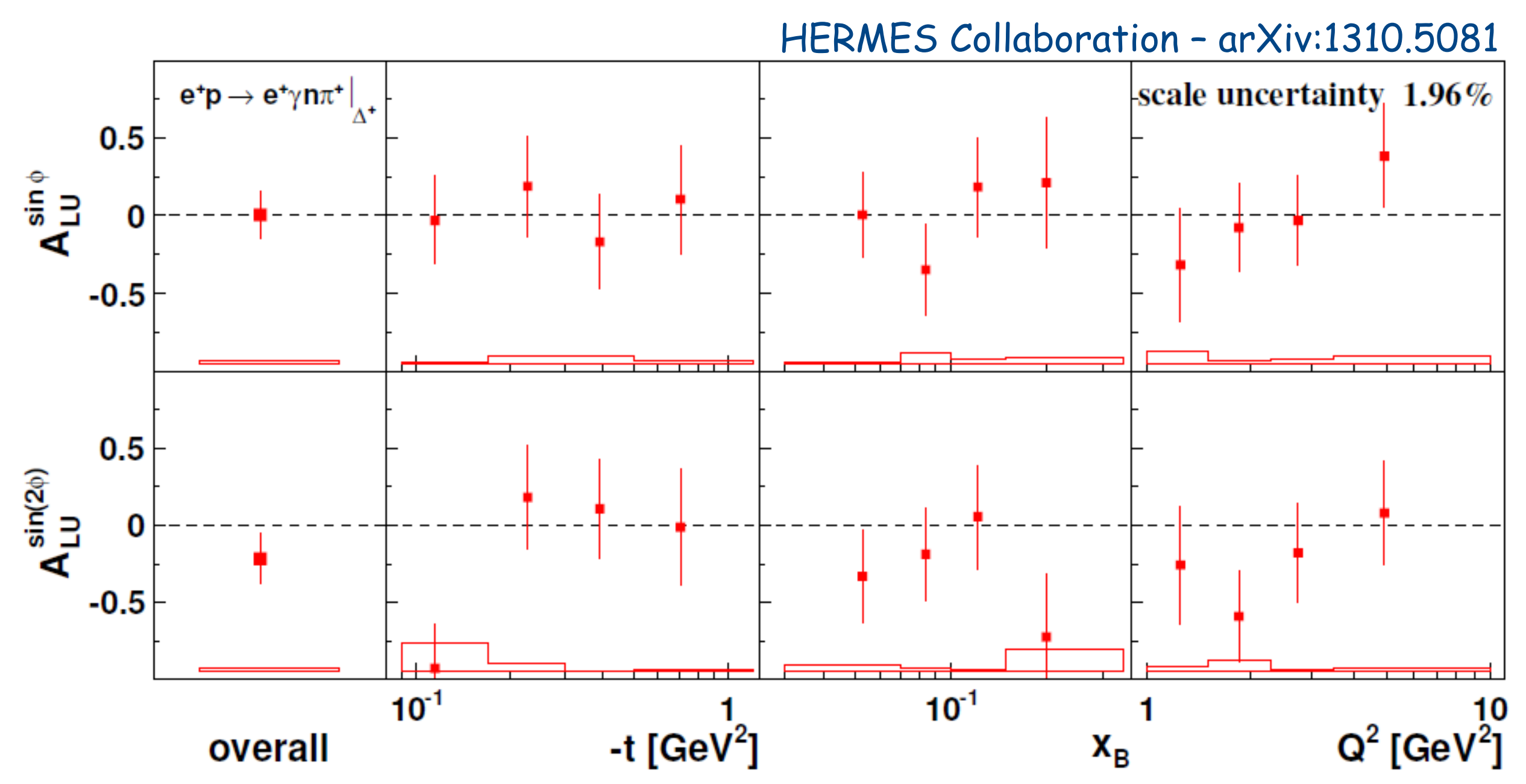

- asymmetry background correction from SIDIS (23\%) and ep $\rightarrow \mathbf{e} \gamma \mathbf{p}(0.2 \%)$

- leading asymmetry consistent with zero 


\section{Exclusive w production}

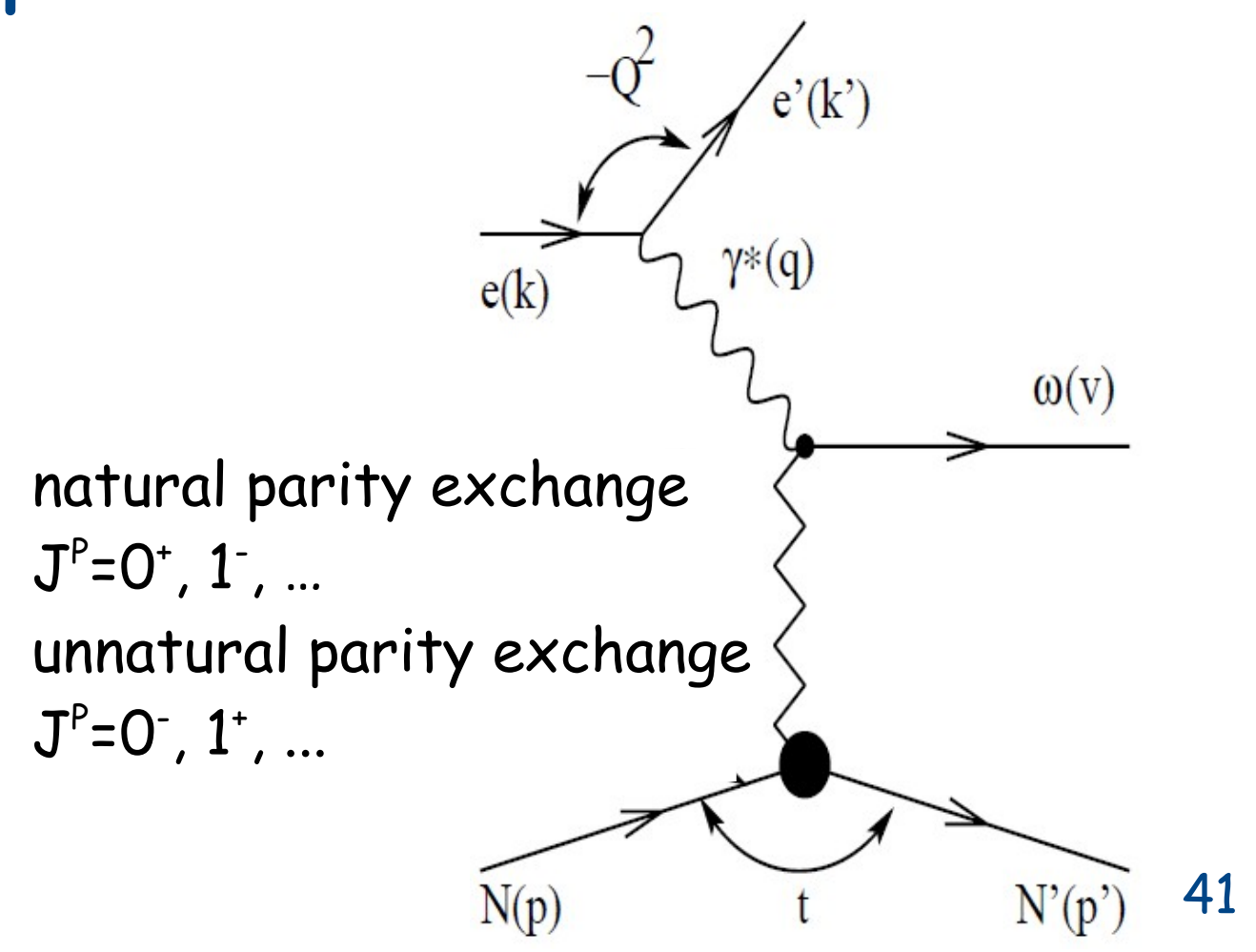




\section{Exclusive $\omega$ production}

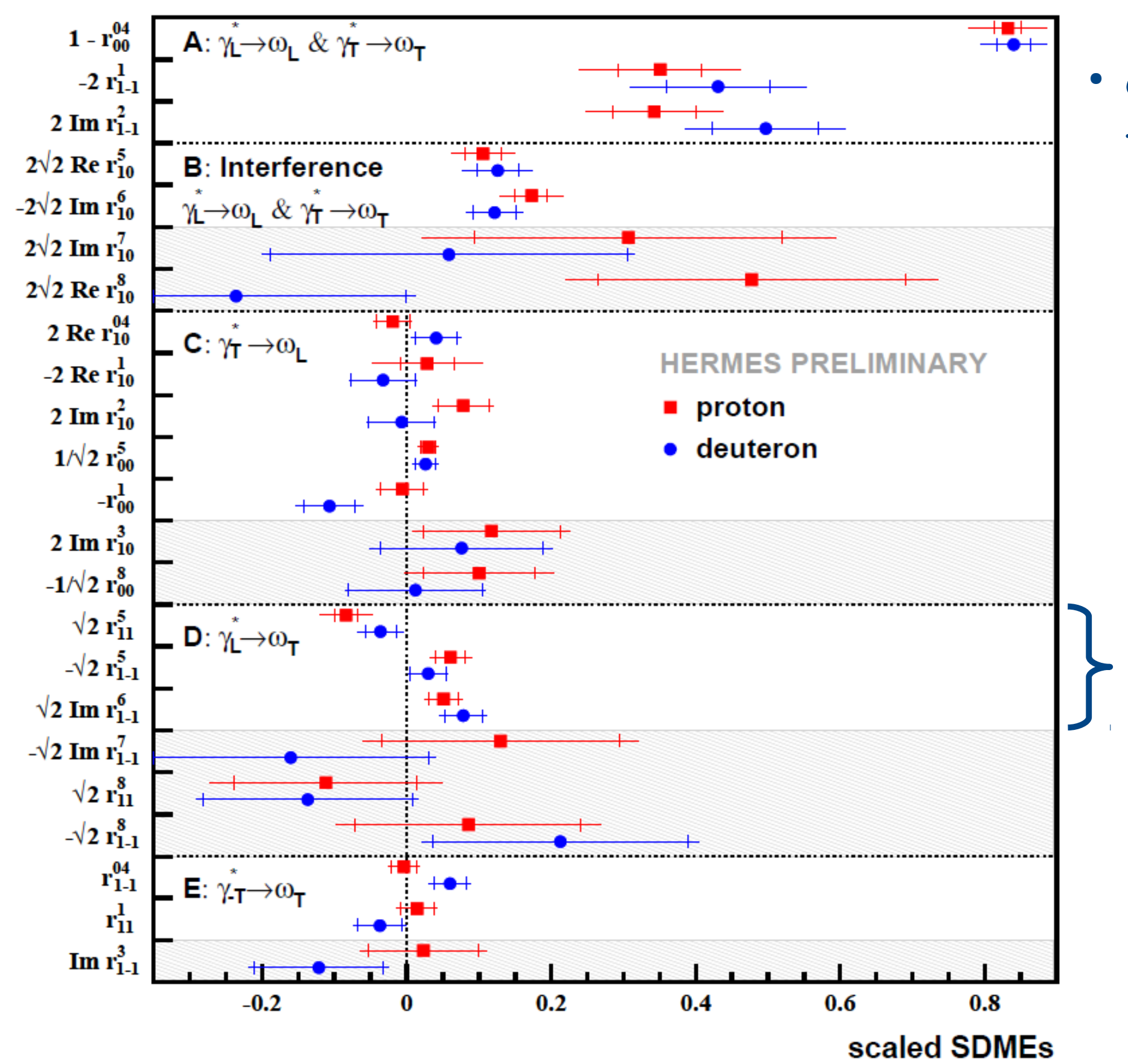

- compatible SDMEs for proton and deuteron

slight violation of s-channel helicity conservation:

$$
\begin{aligned}
& r_{11}^{5}+r_{1-1}^{5}-\Im r_{1-1}^{6} \\
& =-0.14 \pm 0.02 \pm 0.04 \\
& =-0.10 \pm 0.03 \pm 0.03
\end{aligned}
$$




\section{Exclusive $\omega$ production}

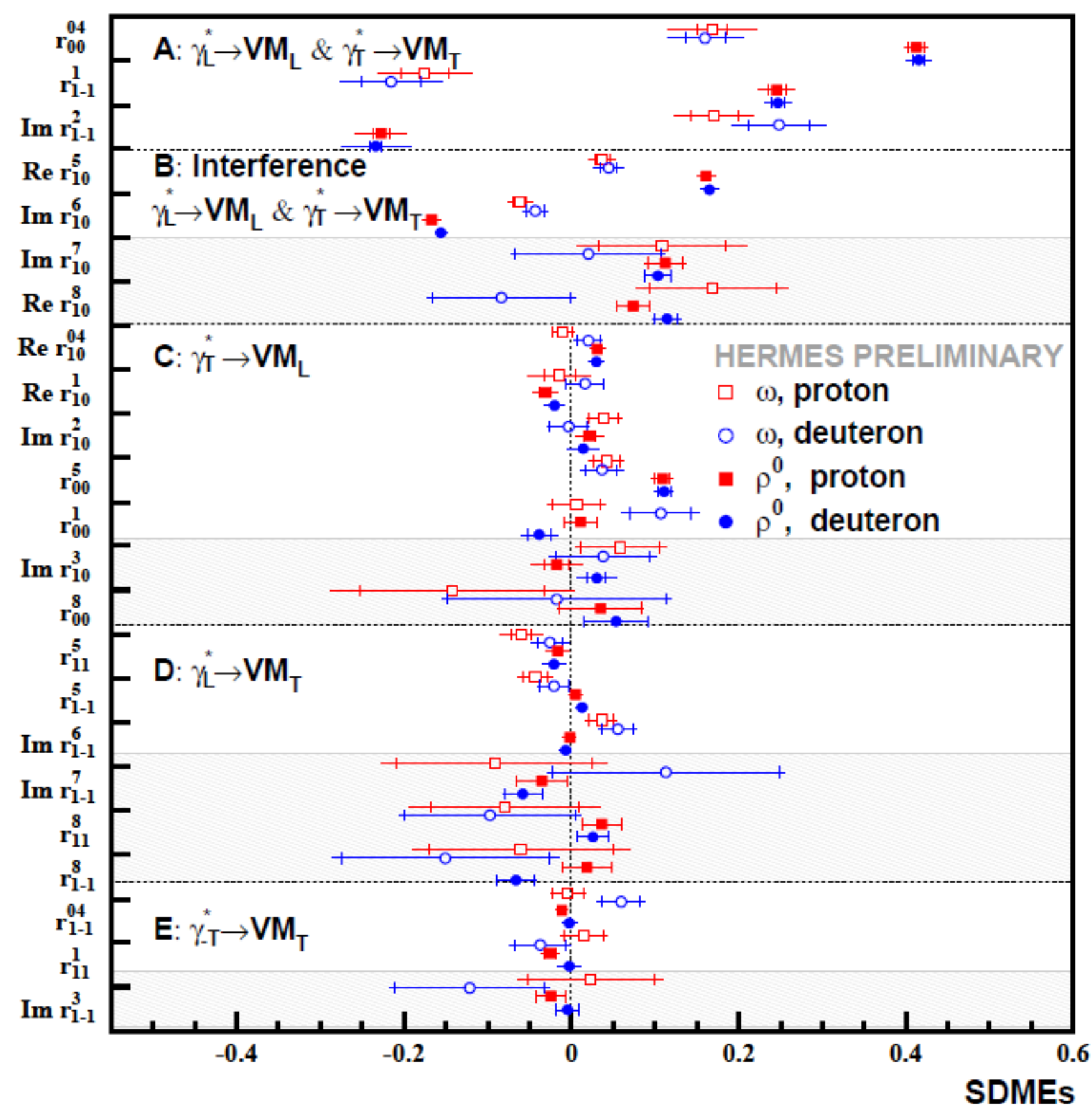

- $\omega-\rho^{0}$ comparison:

- $\omega: \mathbf{r}_{1-1}^{1}<0$

- $\rho^{0}: r_{1-1}^{1}>0$

- $\omega: \Im r_{1-1}^{2}>0$

- $\rho^{0}: \Im \mathbf{r}_{1-1}^{2}<0$

large unnatural parity exchange for $\omega$ production

exclusve $\rho^{0}$ :

A. Airapetian et al., Eur. Phys. J. C 62 (2009) 659 


\section{Exclusive $\omega$ production}

test of unnatural parity exchange:

$$
\begin{aligned}
\mathbf{u}_{\mathbf{1}} & =\mathbf{1}-\mathbf{r}_{\mathbf{0 0}}^{\mathbf{0 4}}+\mathbf{2} \mathbf{r}_{\mathbf{1 - 1}}^{\mathbf{0 4}}-\mathbf{2} \mathbf{r}_{\mathbf{1 1}}^{\mathbf{1}}-\mathbf{2} \mathbf{r}_{\mathbf{1 - 1}}^{\mathbf{1}} \\
& \propto 2 \epsilon\left|U_{10}\right|^{2}+\left|U_{11}+U_{-11}\right|^{2}
\end{aligned}
$$
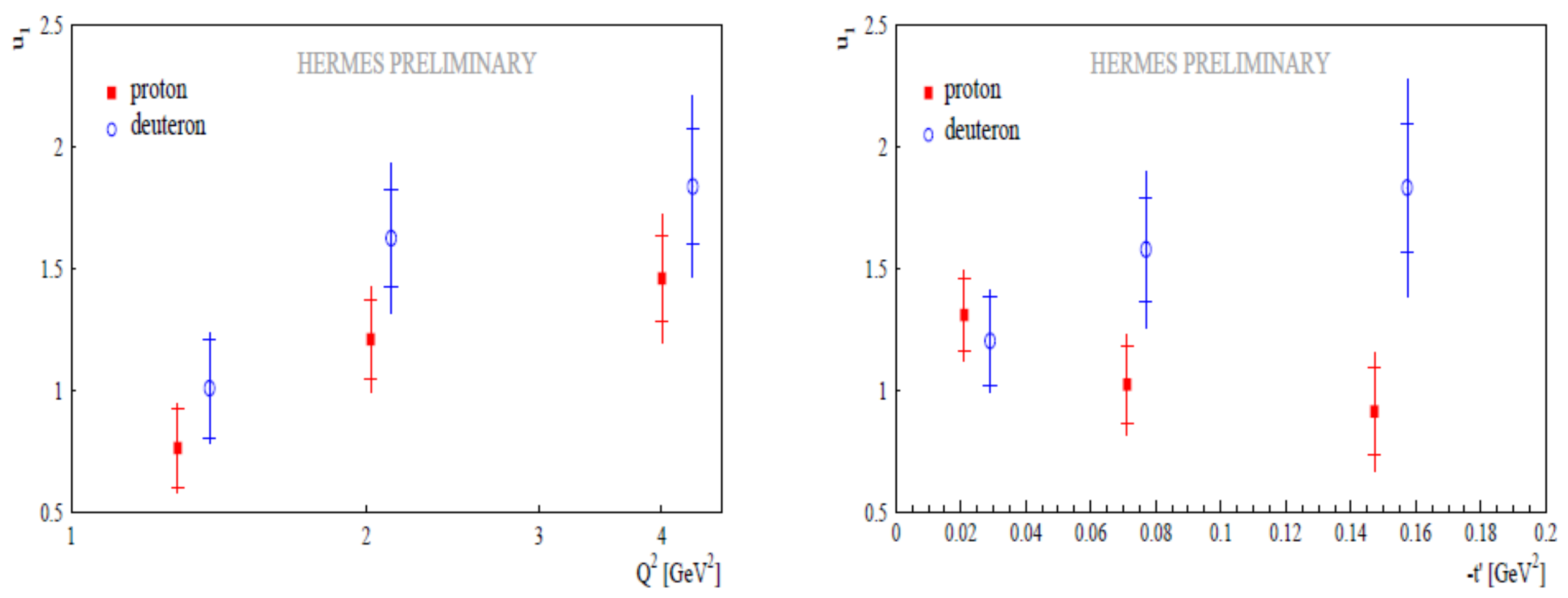

large unnatural-parity exchange 


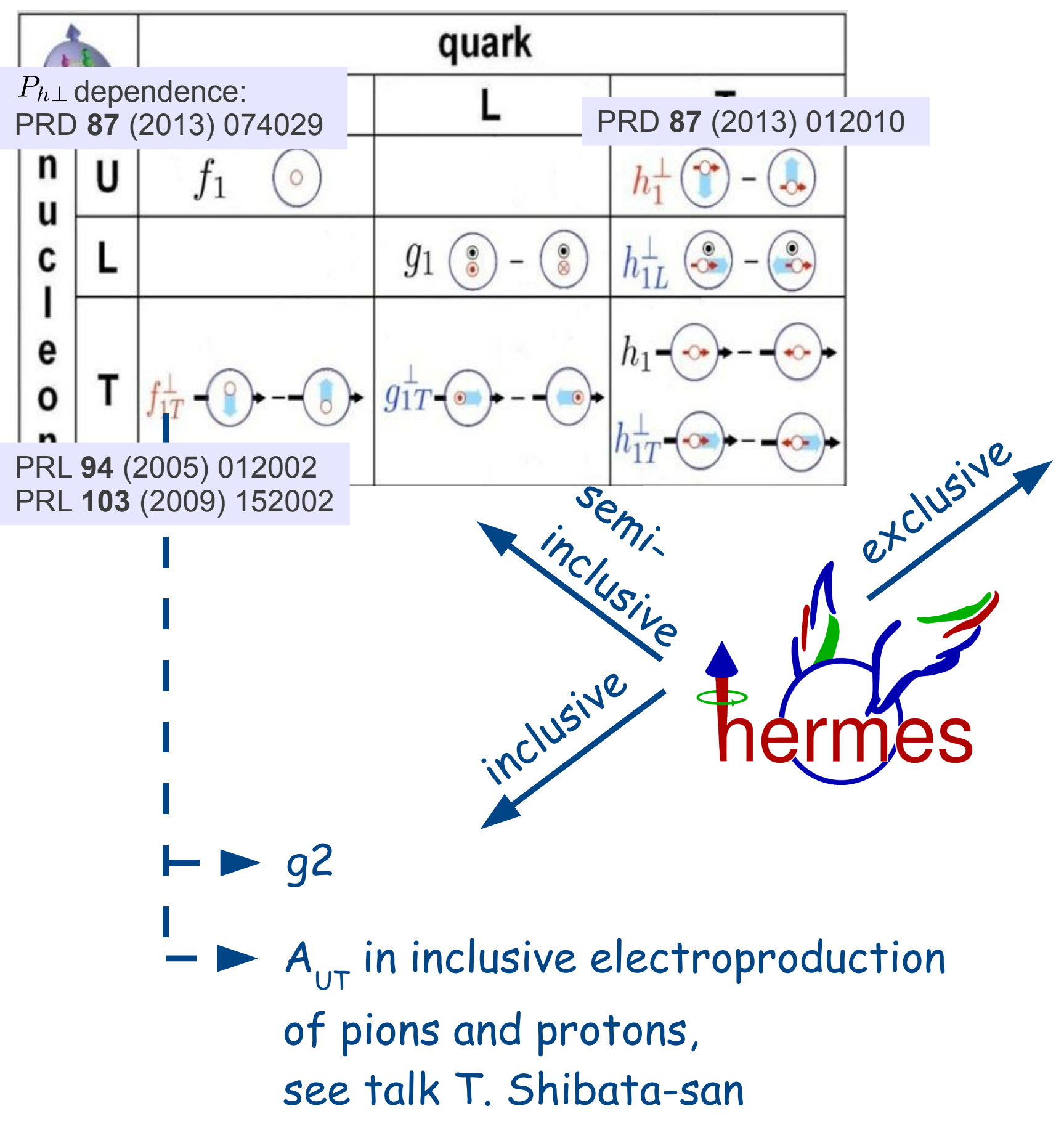

\section{Summary}

exclusive $\omega$ production

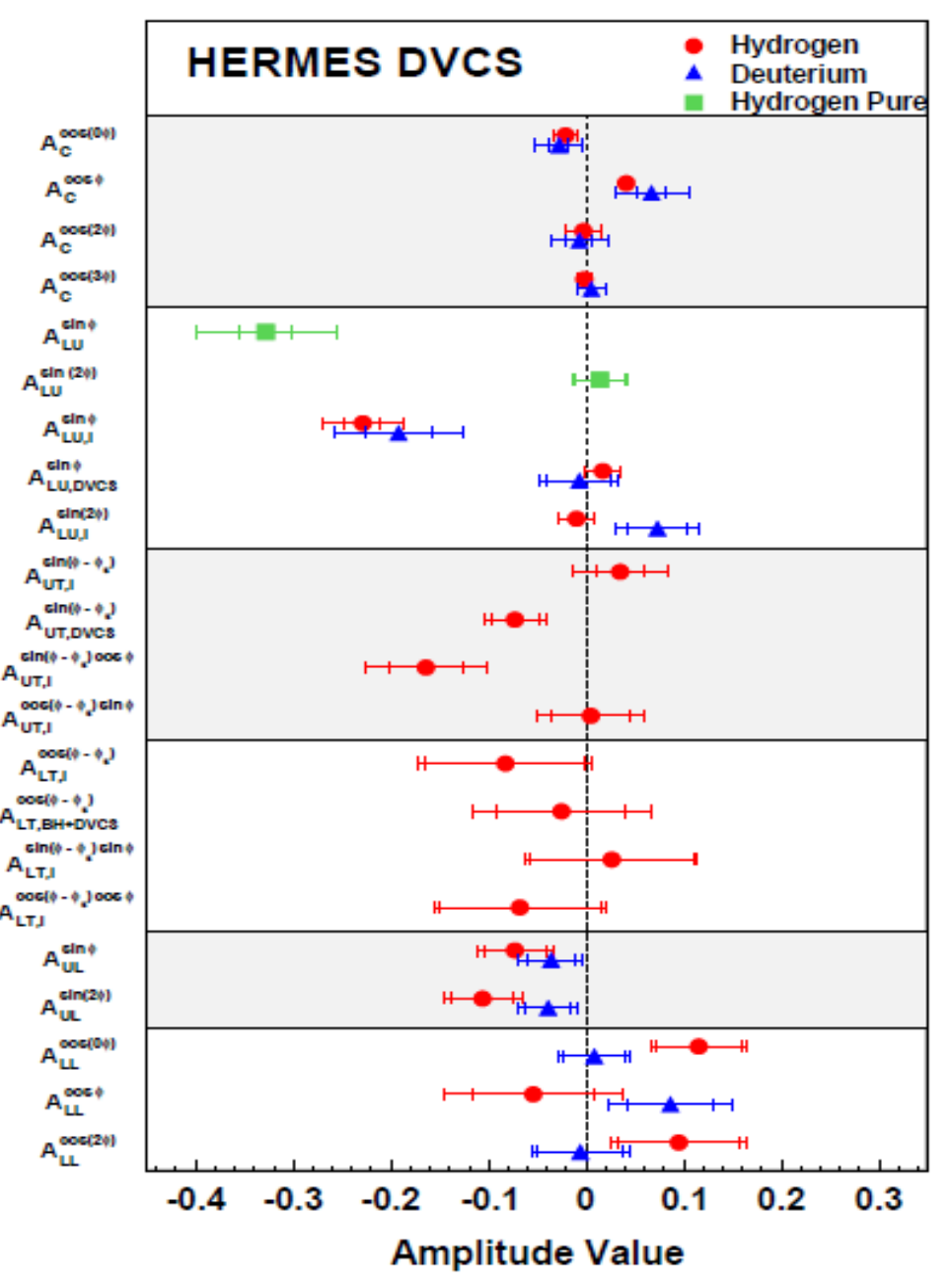




\section{Backup}




\section{Exclusive vector-meson fraction}

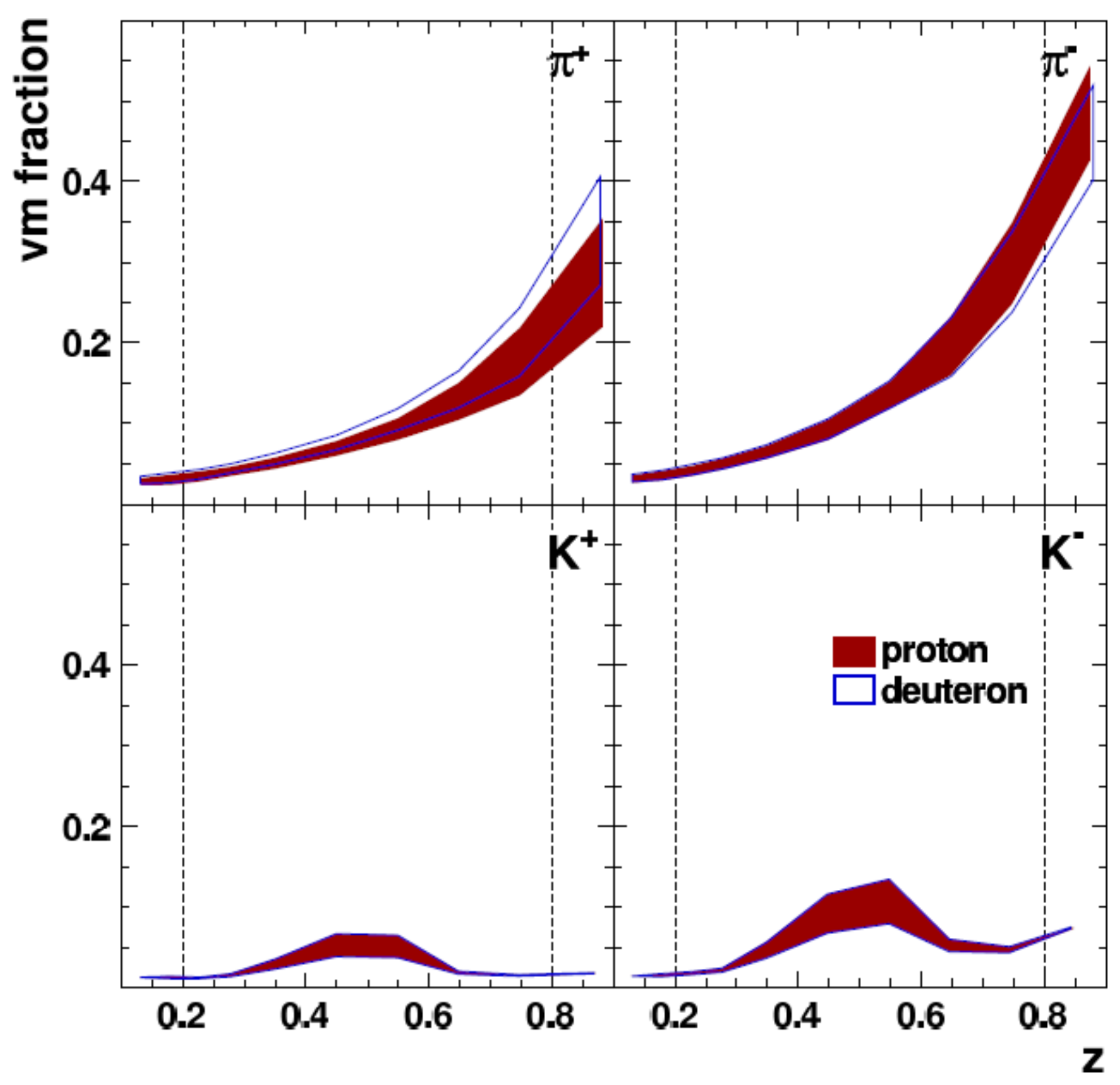


Multiplicities corrected in z: without and with exclusive vector-meson correction

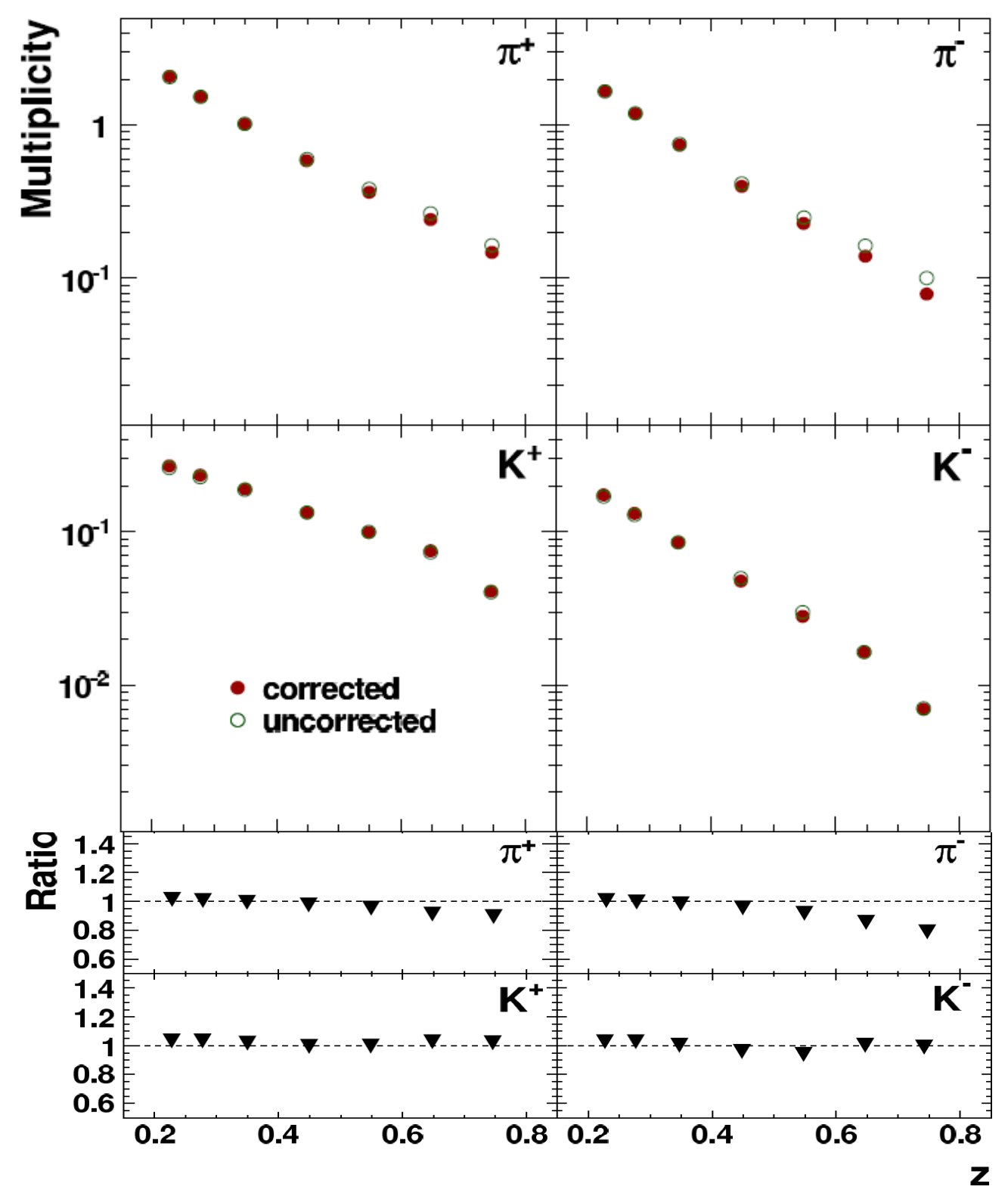




\section{Comparison $S(x)$ with NNPDF21}

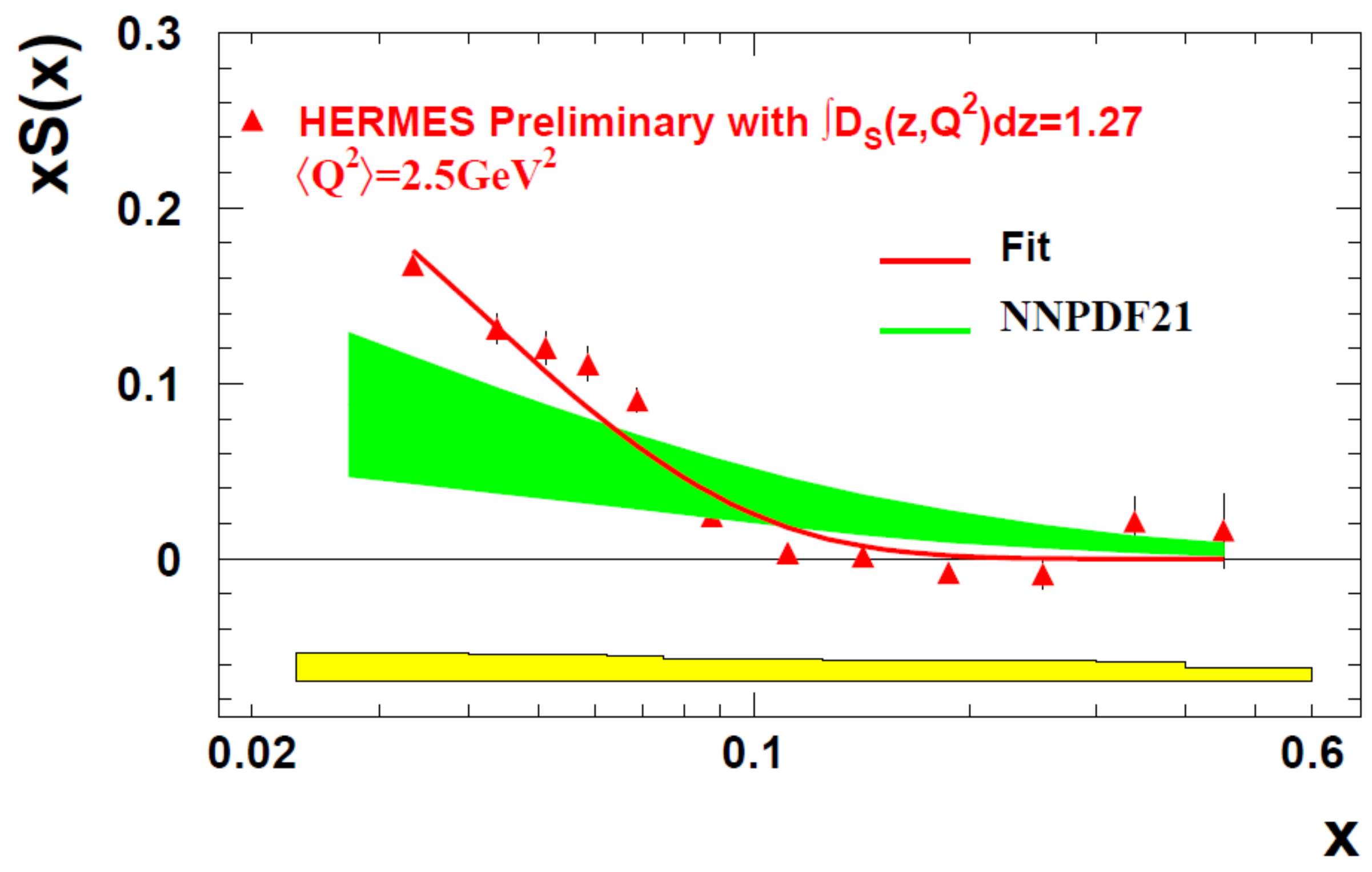




\section{Beam-helicity asymmetry}

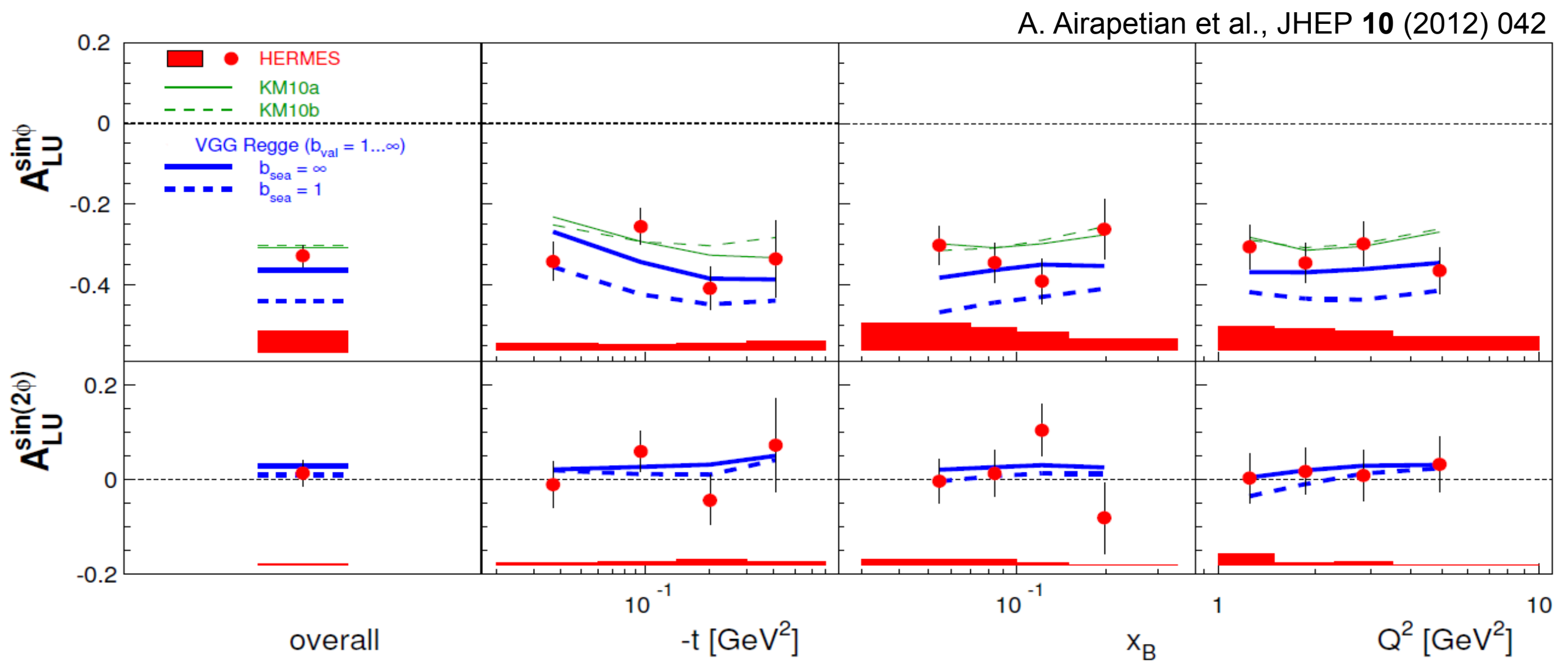

- additional $1.96 \%$ scale uncertainty from beam polarization 


\section{Transverse target single-spin asymmetry in inclusive electroproduction of pions and kaons}

$\mathrm{x}_{\mathrm{F}}=2 \mathrm{P}_{\mathrm{L}} / \sqrt{\mathrm{s}}$ dependence

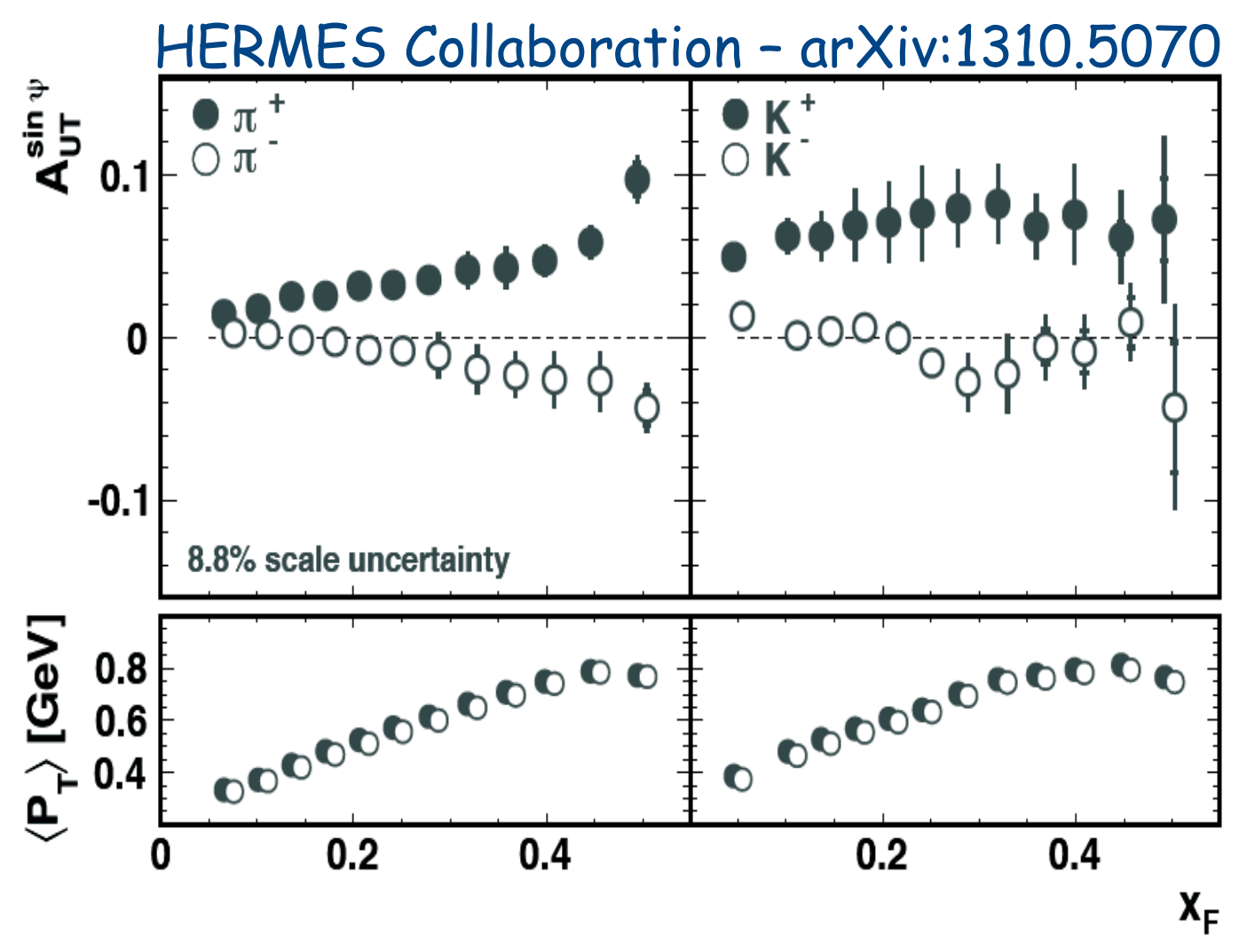

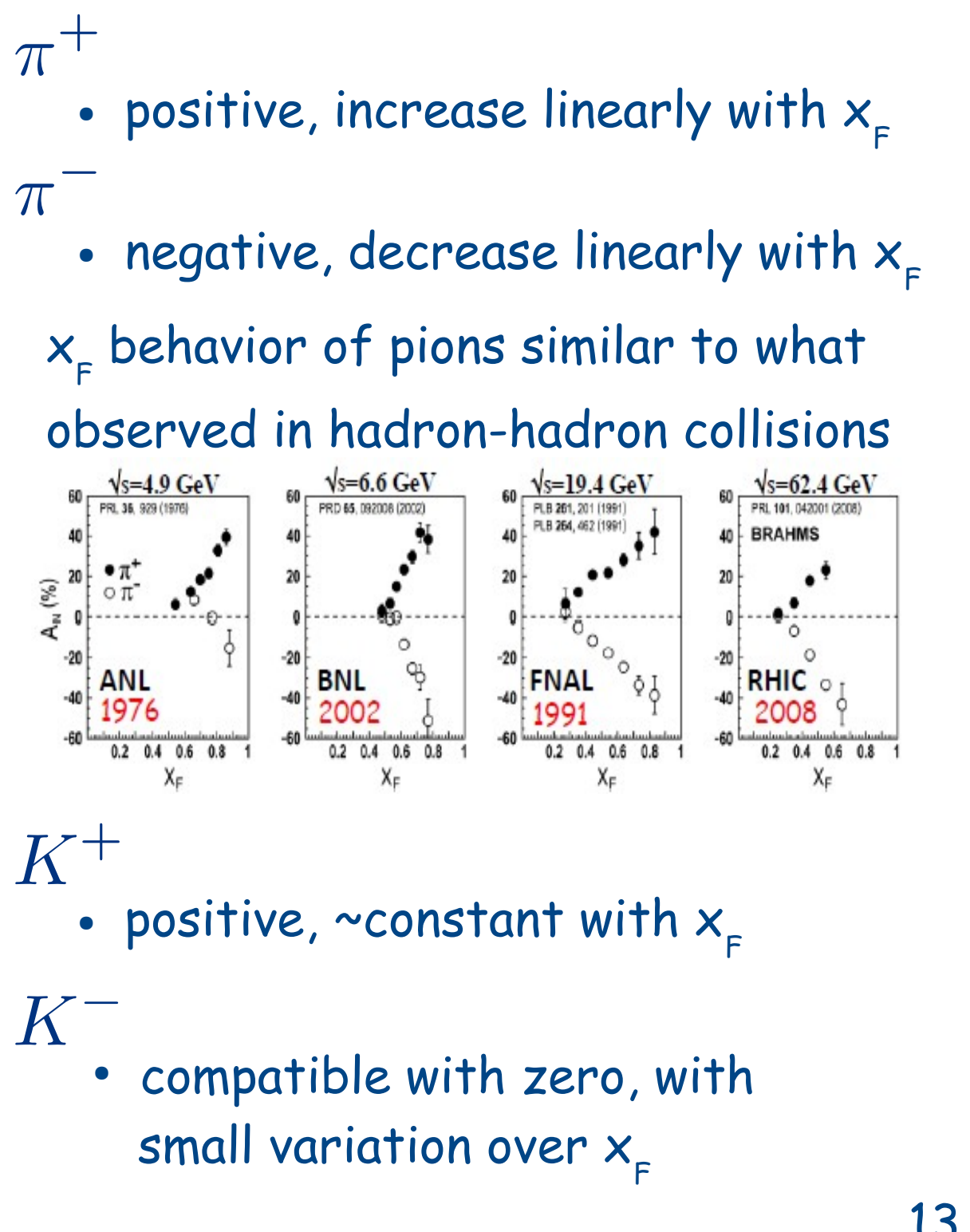




\section{Transverse target single-spin asymmetry in inclusive electroproduction of pions and kaons}

disentangle $\mathbf{X}_{\mathbf{F}}$ and $\mathbf{P}_{\mathbf{T}}$ dependence, HERMES Collaboration-arXiv:1310.5070 $\pi^{+}$

- increase with $P_{T}$ up to $P_{T} \approx 0.8 \mathrm{GeV}$

- $P_{T}$ dependence independent of $x_{F}$ $\rightarrow x_{F}$ increase from $P_{T}$ dependence

$\pi^{-}$

- small amplitudes, varyingly positive and negative with $P_{T}$

- decrease with increasing $x_{F}$ $K^{+}$

- increase with $P_{T}$ up to $P_{T} \approx 0.8 \mathrm{GeV}$

- increase with increasing $X_{F}$

$K^{-}$

- small amplitudes

- decrease with increasing $x_{F}$

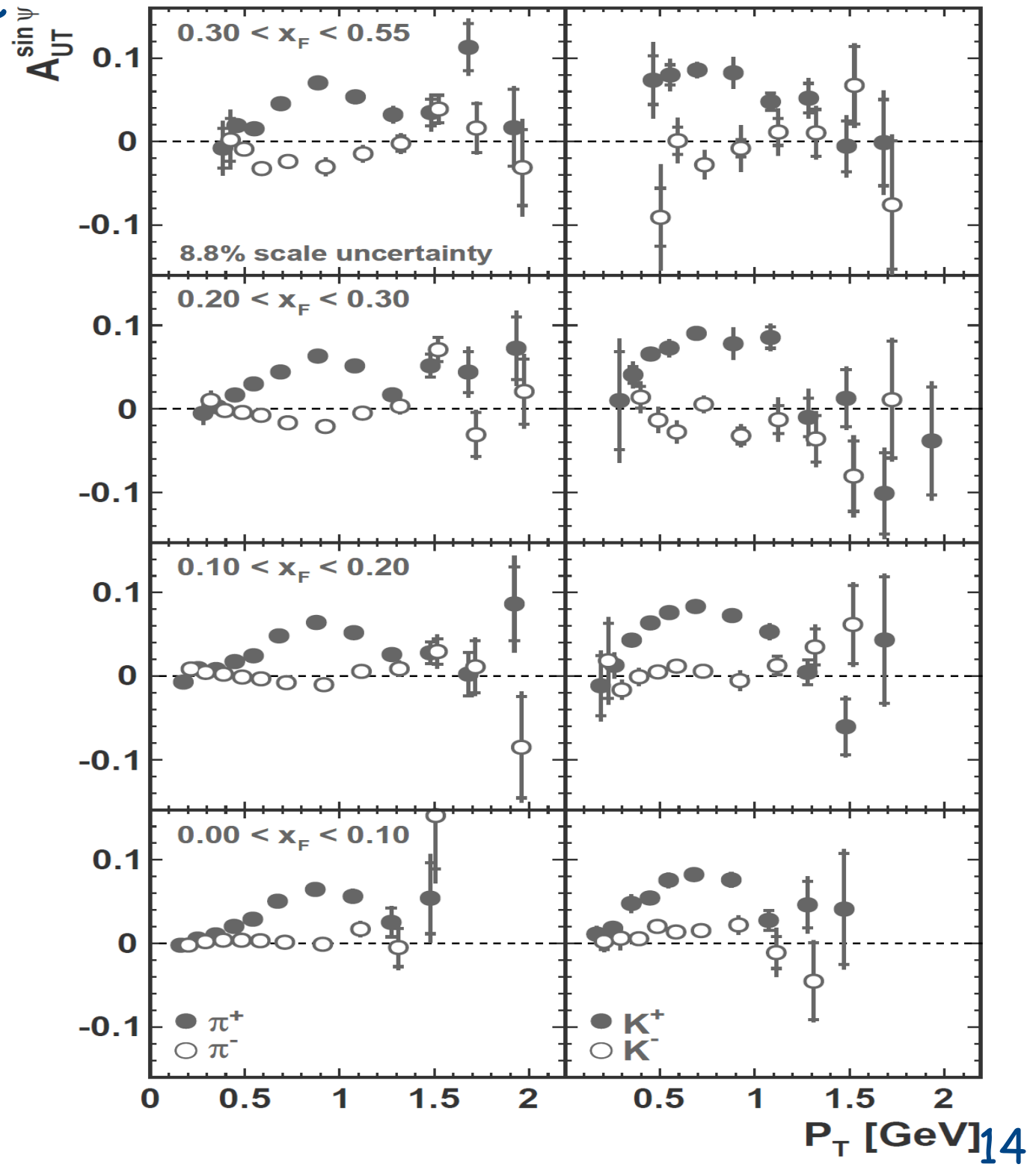

